\title{
Decomposition of nonlocal light-cone operators into harmonic operators of definite twist
}

\author{
Bodo Geyer* \\ Universität Leipzig, Institut für Theoretische Physik \\ and Center for Theoretical Studies \\ Augustusplatz 10, D-04109 Leipzig, Germany \\ Markus Lazar ${ }^{\dagger}$ \\ Universität Leipzig, Institut für Theoretische Physik \\ Augustusplatz 10, D-04109 Leipzig, Germany \\ Dieter Robaschik ${ }^{\ddagger}$ \\ Karl-Franzens-Universität Graz, Institut für Theoretische Physik \\ Universitätsplatz 5, A-8010 Graz, Austria
}

\begin{abstract}
Bilocal light-ray operators which are Lorentz scalars, vectors or antisymmetric tensors, and which appear in various hard QCD scattering processes, are decomposed into operators of definite twist. These operators are harmonic tensor functions and their Taylor expansion consists of (traceless) local light-cone operators which span irreducible representations of the Lorentz group with definite spin $j$ and common (geometric) twist (= dimension - spin). Some applications concerning the nonforward matrix elements of these operators and the generalization to conformal light-cone operators of definite twist is considered. The group theoretical background of the method has been made clear.
\end{abstract}

NTZ 2/99 UNIGRAZ-UTP/27-01-99

hep-th/9901090

PACS: 12.38, 13.88, 02.20; Keywords: Virtual Compton scattering, twist decomposition, nonlocal light-cone operators

*e-mail: geyer@itp.physik.uni-leipzig.de

$\dagger$ e-mail: lazar@itp.physik.uni-leipzig.de

${ }^{\ddagger}$ present address: DESY-IfH Zeuthen, Platanenallee 6, 15735 Zeuthen 


\section{Introduction}

For many hard scattering processes in QCD the nonlocal light-cone(LC) expansion, together with the renormalization group equation, is a powerful tool to determine the dependence of the nonperturbative partition amplitudes on the experimentally relevant momentum transfer $Q^{2}$. This is the case for, e.g., the parton distributions in deep inelastic scattering (DIS), the pion as well as vector meson wave functions and, as growing up more recently, the non-forward distribution amplitudes in deeply virtual Compton scattering (DVCS). Any of these phenomenological quantities are related to the Compton amplitude for non-forward scattering a virtual photon off a hadron which is given by

$$
T_{\mu \nu}\left(p_{+}, p_{-}, Q\right)=i \int d^{4} x e^{i q x}\left\langle p_{2}, S_{2}\left|T\left(J_{\mu}(x / 2) J_{\nu}(-x / 2)\right)\right| p_{1}, S_{1}\right\rangle
$$

where $p_{+}=p_{2}+p_{1}, p_{-}=p_{2}-p_{1}=q_{1}-q_{2}, q=\left(q_{1}+q_{2}\right) / 2$ are the kinematical independent variables. To specify the asymptotics of virtual Compton scattering the generalized Bjorken region will be defined as follows

$$
\nu=q p_{+} \longrightarrow \infty, \quad Q^{2}=-q^{2} \longrightarrow \infty
$$

with the two independent scaling variables $\xi=Q^{2} / q p_{+}$and $\xi^{\prime}=q p_{-} / q p_{+}=$ $\left(q_{1}^{2}-q_{2}^{2}\right) / 2 \nu$, to be held fixed by the experimental setup. These variables which are not restricted to the intervall $[-1,+1]$ are obvious modifications of the usual Bjorken variable and the 'skewedness' parameter.'

The (renormalized) time-ordered product in eq. (1.1) can be represented in terms of the nonlocal operator product expansion [2, 3, 27], which directly leads to compact expressions for the coefficient functions and the corresponding bilocal LC-operators. It is essential to remark that all the interesting nonperturbative partition amplitudes which appear in the above mentioned processes are Fourier transforms of various matrix elements whose unique input are one and the same light-ray operators. Furthermore, all the interesting evolution equations of these partition amplitudes result from the renormalization group equation of the involved light-ray operators and, consequently, their evolution kernels can be determined from the anomalous dimension of these operators. So many of the physical properties which result in different experimental situations have a common quantum field theoretical origin which is traced back to the properties of the LC-operators.

With the growing accuracy of the experimental data a perturbative expansion with respect to the experimentally relevant variable, $M^{2} / Q^{2}, M$ being a typical mass of the process, will be very useful. However, these contributions could be of quite different origin. On the one hand, they result from a decomposition of the bilocal light-ray operators - in lowest order only quark operators with two

${ }^{1}$ Instead of this parametrization, despite being quite natural from a general point of view, for various physical situations others may be prefered (see, e.g., [22, 30, 21, 17]). 
external legs occure - into operators of definite twist. In addition there appear higher loop contributions of physical matrix elements for the same operators.

Unfortunately, the notion of twist is unambigously defined in leading order only. Its original definition, as has been introduced by Gross and Treiman 16] for the local operators, twist $(\tau)=$ dimension $(d)$ - spin $(j)$, is directly related to the irreducible tensor representations of the orthochronous Lorentz group and, with respect to this, is a Lorentz invariant notion ("geometric twist").

Later on, various authors used the well known LC-quantization in the infinite momentum frame [24] together with the decomposition of the quark fields into "good" and "bad" components, $\psi=\psi_{+}+\psi_{-}$, with $\psi_{ \pm}=\frac{1}{2} \gamma^{\mp} \gamma^{ \pm} \psi$, in order to introduce another notion of twist: As has been pointed out in [20], a "bad" component introduces one unit of $M / Q$ because these components are not dynamically independent and may be expressed through the equations of motion by the "good" ones times the above kinematical factor. Despite being conceptionally different this definition looks similar to the phenomenologically more convenient one which counts only powers of $1 / Q$ in the infinite momentum frame [20].

However, both these power-counting concepts of "dynamical twist", despite being phenomenological quite useful, have serious theoretical drewbacks: First, they are not Lorentz invariant and, therefore, can not coincide with the original geometric definition. Furthermore, the same power of $1 / Q$ may occure for different values of $\tau$. In addition, this concept is applicable only for matrix elements of operators and not directly related to the operators itself. Moreover, if the matrix elements contain more than one momentum, as will be the case for DVCS, the definition of the scaling variables is somewhat ambiguous and different definitions are related by power series in $M^{2} / Q^{2}$ thereby changing the decomposition with respect to "dynamical twist".

Therefore, from the point of view of renormalized quantum field theory we should state: Only the original geometric definition of twist is a well-defined concept. But then the problem occurs how to generalize it to the nonlocal operators which appear in the nonlocal light-cone expansion.

Let us shortly introduce the basic concepts of the nonlocal light-cone expansion and show how it is related to its local version. The rigorous proof of the nonlocal LCE was given in [2] where, first of all, an operator identity for the product of two composite operators - e.g. the (time ordered) product of the two currents in eq. (1.1) - has been proven (in scalar field theory) which holds on the whole Hilbert space. Then, using a special, light-cone adapted renormalization procedure $R$ it was shown that the perturbative functional of the renormalized product of two currents may be split into an asymptotically relevant part - the light-cone expansion up to a finite order of light-cone singularity - and a welldefined remainder being less singular:

$$
\begin{gathered}
R T\left(J_{\mu}(x / 2) J_{\nu}(-x / 2) S\right) \approx \int_{-1}^{+1} d^{2} \underline{\kappa} C_{\Gamma}\left(x^{2}, \underline{\kappa} ; \mu^{2}\right) R T\left(O^{\Gamma}\left(\kappa_{1} \tilde{x}, \kappa_{2} \tilde{x}\right) S\right) \\
+ \text { higher order terms. }
\end{gathered}
$$


Here, the (unrenormalized) light-ray operators are given by

$$
O^{\Gamma}\left(\kappa_{1} \tilde{x}, \kappa_{2} \tilde{x}\right)=: \bar{\psi}\left(\kappa_{1} \tilde{x}\right) \Gamma U\left(\kappa_{1} \tilde{x}, \kappa_{2} \tilde{x}\right) \psi\left(\kappa_{2} \tilde{x}\right):
$$

with some specified $\Gamma$-structure and with the usual phase factor

$$
U\left(\kappa_{1}, \kappa_{2}\right) \equiv U\left(\kappa_{1} \tilde{x}, \kappa_{2} \tilde{x}\right)=\mathcal{P} \exp \left\{-i g \int_{\kappa_{2}}^{\kappa_{1}} d \tau \tilde{x}^{\mu} A_{\mu}(\tau \tilde{x})\right\},
$$

where $\mathcal{P}$ denotes the path ordering, $g$ is the strong coupling parameter and $A_{\mu}=$ $A_{\mu}^{a} t^{a}$ is the gluon field with $t^{a}$ being the generators of $S U(3)_{\text {color }}$ in the fundamental representation spanned by the quark fields $\psi$. Thereby, the light-like vector

$$
\tilde{x}=x+\eta\left(x \eta / \eta^{2}\right)\left(\sqrt{1-x^{2} \eta^{2} /(x \eta)^{2}}-1\right)
$$

is related to $x$ and a fixed non-null subsidiary four-vector $\eta$ whose dependence drops out in physical expressions at leading order. In this approach the coefficient functions and the corresponding light-ray operators naturally occure as renormalized ones. In the following, for simplicity, we do not explicitly indicate that they are renormalized quantities.

Let us now point to the remarkable fact that, in principle, the nonlocal as well as the usual local light-cone expansion may be obtained from (1.3) (for a detailed consideration see [10, 12]): The Fourier transform $F_{\Gamma}\left(x^{2}, \tilde{x} p_{i} ; \mu^{2}\right)$ of $C_{\Gamma}\left(x^{2}, \kappa_{i} ; \mu^{2}\right)$ with respect to $\kappa_{i}$ is an entire functions of the new arguments $\tilde{x} p_{i}$ [2]. Therefore, the range of $\kappa_{i}$ is restricted to $-1 \leq \kappa_{i} \leq+1$ and, in addition, $F_{\Gamma}$ may be Taylor expanded with respect to $\tilde{x} p_{i}$ : this leads, instead of the $\kappa$-integration in (1.3), to a (twofold) infinite sum over local operators. The local and nonlocal operators are related through the following formulae:

$$
\begin{aligned}
O^{\Gamma}\left(\kappa_{1} \tilde{x}, \kappa_{2} \tilde{x}\right) & =\sum_{n_{1} n_{2}} \frac{\kappa_{1}^{n_{1}}}{n_{1} !} \frac{\kappa_{2}^{n_{2}}}{n_{2} !} O_{n_{1} n_{2}}^{\Gamma}(\tilde{x}), \\
O_{n_{1} n_{2}}^{\Gamma}(\tilde{x}) & =\left.\frac{\partial^{n_{1}}}{\partial \kappa_{1}^{n_{1}}} \frac{\partial^{n_{2}}}{\partial \kappa_{2}^{n_{2}}} O^{\Gamma}\left(\kappa_{1} \tilde{x}, \kappa_{2} \tilde{x}\right)\right|_{\kappa_{1}=\kappa_{2}=0}
\end{aligned}
$$

This connection between the local and the nonlocal LC-operators is the key relation which opens the possibility to generalize the geometric notion of twist, being originally introduced for local operators, also to nonlocal ones. This is the aim of the present paper which is essentially based on [26, 8]. At first, a group theoretical founded algorithm is presented which allows to obtain for any bilocal operator a uniquely defined twist decomposition

$$
O_{\Gamma}\left(\kappa_{1} x, \kappa_{2} x\right)=\sum_{\tau_{\min }}^{\infty} O_{\Gamma}^{\tau}\left(\kappa_{1} x, \kappa_{2} x\right) .
$$

\footnotetext{
${ }^{2}$ It has to be noted that, because the light-cone expansion is considered for the operators and not for their matrix elements, such a light-like reference vector has necessarily to be introduced in configuration space. However, if matrix elements are considered with some uniquely defined momentum $P, P^{2}=M^{2}$, then $\eta=P / M$ may be chosen [6].
} 
For general values of $x$ this decomposition contains infinitely many terms which are harmonic tensor functions (depending on the "external" Lorentz indices $\Gamma$ ). In general, the contributions to a well-defined twist result from various independent irreducible (local) tensor representations. By projecting onto the light-cone, $x \rightarrow \tilde{x}$, the above sum terminates at finite twist $\tau_{\max }$. For the bilocal quark operators, which will be considered in detail, we obtain $\tau_{\max }=4$. For the vector and (antisymmetric) tensor operators the trace terms which define operators of higher twist are proportional to $\tilde{x}_{\beta}$. Their explicit form has been given here for the first time. Of course, the method can be applied to the gluon operators; in principle, also more complicated nonlocal operators may be considered.

It should be mentioned here that a special version of that programme making extensive use of Schwinger-Fock gauge for the quark string operators, however without unravelling the explicit symmetry type behind such twist decompositions, already was given in Ref. [4]. In addition we remark that a twist decomposition of special conformal operators was given in Ref. [14] [ thereby taking profit from the properties of conformal invariant 3-point functions of (generalized) free fields.

The paper is organized as follows. Section 2 is devoted to a short exposition of the group theoretical background characterizing the irreducible tensor representations of the Lorentz group. Section 3 contains our main results - the twist decomposition of (pseudo) scalar, (axial) vector and antisymmetric tensor operators. Appendix A contains a short exposition about the characterization of irreducible tensor representations through the Young tableaux', and Appendix B introduces the notion of harmonic tensor functions which is relevant for our twist decomposition.

\section{Irreducible tensor representations of the Lorentz group $\mathcal{L}_{+}^{\uparrow} \simeq S O(1,3, \mathbb{R})$}

In this Chapter the group theoretical background will be given which is used for the construction of the nonlocal operators whose local parts - which are obtained by Taylor expansion - transform according to irreducible tensor representations under the Lorentz group, i.e., have definite spin and therefore also well defined twist. These tensor representations are characterized by a specific symmetry class with respect to the symmetric group and are determined by a few types of Young tableaux.

The Lie algebra of the Lorentz group $\mathcal{L}_{+}^{\uparrow}$ is characterized by the generators of the three spatial rotations $\vec{M}$ and the three boosts $\vec{N}$; from it the two (complex) linear combinations $\vec{M}_{ \pm}$may be build which define two independent $S O(3)$ groups:

$$
\left[M_{ \pm}^{i}, M_{ \pm}^{j}\right]=i \epsilon^{i j k} M_{ \pm}^{k}, \quad\left[M_{+}^{i}, M_{-}^{j}\right]=0 \quad \text { with } \quad \vec{M}_{ \pm}=\vec{M} \pm i \vec{N}
$$

Therefore, the (Lie algebra of the) complex Lorentz group is isomorphic to the

\footnotetext{
${ }^{3}$ The authors are much indebted to D. Müller for bringing that referenc to their attention.
} 
(Lie algebra of the) direct product $S O(3, \mathbb{C}) \otimes S O(3, \mathbb{C}) \simeq S O(4, \mathbb{C})$. This characterization makes use of the fact that the irreducible (finite) representations

$$
\mathcal{D}^{\left(j_{+}, j_{-}\right)}(\vec{\varphi}, \vec{\vartheta})=\mathcal{D}^{\left(j_{+}\right)}(\vec{\varphi}-i \vec{\vartheta}) \otimes \mathcal{D}^{\left(j_{-}\right)}(\vec{\varphi}+i \vec{\vartheta})
$$

of the restricted orthochronous Lorentz group $\mathcal{L}_{+}^{\uparrow} \simeq S O(1,3 ; \mathbb{R})$, where $\vec{\varphi}$ and $\vec{\vartheta}$ are the angle of rotation and the rapidity of the boost transformations, are determined by two numbers $\left(j_{+}, j_{-}\right), j_{ \pm}=0,1 / 2,1, \cdots$, which define the spin $j=j_{+}+j_{-}$of the representation.

Let us now state some general results concerning finite dimensional representations of the (complex) orthogonal groups (see, e.g., Chapters 8 and 10 of Ref. [7]): (1) The group $S O(N, \mathbb{C})$ has two series of complex-analytif irreducible representations. Every representation of the first (resp. the second) series determines and is in turn determined by a highest weight $\underline{m}=\left(m_{1}, m_{2}, \ldots, m_{\nu}\right)$ whose components $m_{i}$ are integers (resp. half-odd integers) and satisfy the conditions

$$
\begin{aligned}
m_{1} \geq m_{2} \geq \ldots \geq m_{\nu-1} \geq\left|m_{\nu}\right| & \text { for } \quad N=2 \nu \\
m_{1} \geq m_{2} \geq \ldots \geq m_{\nu-1} \geq m_{\nu} \geq 0 & \text { for } \quad N=2 \nu+1 .
\end{aligned}
$$

The first series determines the tensor representations, whereas the second series determines the spinor representations.

(2) A tensor representation of $S O(N ; \mathbb{C})$, for either $N=2 \nu$ or $N=2 \nu+1$, being determined by the highest weight $\underline{m}=\left(m_{1}, m_{2}, \ldots, m_{\nu}\right), m_{i}$ integer, is equivalent to another tensor representation $T_{i_{1} i_{2} \ldots i_{n}}, n=\sum m_{i}$, which is realized in the space of traceless tensors whose symmetry class is characterized by a Young pattern defined by the partition $[m]=\left(m_{1}, m_{2}, \ldots, m_{\nu}\right)$.

(3) Furthermore, if $\mathcal{T}_{G_{c}}$ is a complex-analytic representation of a (complex) semi-simple Lie group $G_{c}$ and $\mathcal{T}_{G_{r}}$ is the restriction of $\mathcal{T}_{G_{c}}$ to a real form $G_{r}$ of $G_{c}$, then, $\mathcal{T}_{G_{c}}$ is irreducible (fully reducible) iff $\mathcal{T}_{G_{r}}$ is irreducible (fully reducible). As a consequence, any irreducible representation of the complex group remains irreducible if restricted to a real subgroup and, on the other hand, from any irreducible representation of the real group by analytic continuation in the group parameters an irreducible representation of its complexification is obtained.

Therefore, in order to investigate irreducible tensor representations of the Lorentz group we may consider equally well irreducible tensor representations of the complex group $S O(4, \mathbb{C})$. Even more, any irreducible representation of the orthogonal group $S O(4)$ by analytic continuation induces an irreducible representation of $S O(4, \mathbb{C})$, which by restriction to their real subgroup $\mathcal{L}_{+}^{\uparrow}$ subduces an irreducible representation of the Lorentz group. Since tensor representations of the orthogonal group are uniquely determined by the symmetry class $[m]$ of their tensors we may study the irreducible tensor representations of the Lorentz group through a study of the corresponding Young tableaux which determine the irreducible representations of the symmetric group $S_{n}$ of permutations.

\footnotetext{
${ }^{4}$ A representation is complex-analytic if it depends analytically on the group parameters.
} 
This characterization of tensor representations through their symmetry class holds for any of the classical matrix groups, $G L(N, \mathbb{C})$ and their various subgroups (see also Appendix A). However, for $O(N, \mathbb{C})$ and $S O(N, \mathbb{C})$ these representations, in general, are not irreducible. The reason is that taking the trace of a tensor commutes with the orthogonal transformations. Therefore, by Schur's Lemma, irreducible subspaces of $S O(N, \mathbb{C})$ are spanned by traceless tensors having definite symmetry class.

From the requirement of tracelessness the following restrictions obtain (see, e.g., [18]): The only Young tableaux' being relevant are those whose first two columns are restricted to have lenght $\mu_{1}+\mu_{2} \leq N$ (see (i) - (iv) below). Two representations $R$ and $R^{\prime}$ whose first columns are related by $\mu_{1}^{\prime}=N-\mu_{1}$, where $\mu_{1} \leq N / 2$, are called associated; if $\mu_{1}=\mu_{1}^{\prime}=N / 2$ this representation is called selfassociated. After restriction to the subgroup $S O(N) \subset O(N)$ associated representations are equivalent, whereas selfassociated representations decompose into two nonequivalent irreducible representations.

Let us illustrate this for the Lorentz group by the representations which will be of interest in the following. First of all, vector and axial vector representations $V_{\mu}$ and $A_{\mu}=\epsilon_{\mu \nu \kappa \lambda} A^{\nu \kappa \lambda}$, respectively, are associated ones, and antisymmetric tensor representations, $A_{\mu \nu}=T_{\mu \nu}-T_{\nu \mu}$, are selfassociated which, if restricted to $S O(1,3)$ decompose into the (anti-)selfdual tensors $A_{\mu \nu}^{ \pm}=\frac{1}{2}\left(A_{\mu \nu} \mp \frac{1}{2} \mathrm{i} \epsilon_{\mu \nu \kappa \lambda} A^{\kappa \lambda}\right)$. Since later on only representations of the orthochronous Lorentz group containing the parity operation are considered, this distinction is of no relevance. Therefore, any tensor of second order, $T_{\mu \nu}$, may be decomposed according to

$$
T_{\mu \nu}=\underbrace{\frac{1}{2}\left(T_{\mu \nu}+T_{\nu \mu}\right)-\frac{1}{4} g_{\mu \nu} T_{\rho}^{\rho}}_{S_{\mu \nu}}+\underbrace{\frac{1}{2}\left(T_{\mu \nu}-T_{\nu \mu}\right)}_{A_{\mu \nu}}+\frac{1}{4} g_{\mu \nu} T_{\rho}^{\rho} .
$$

Let us denote by $\mathbf{T}\left(j_{+}, j_{-}\right), j_{+}+j_{-}$integer, the space of tensors which carry an irreducible representation $\mathcal{D}^{\left(j_{+}, j_{-}\right)}$of the Lorentz group. Then from eq. (2.4) we read off: $S_{\mu \nu} \in \mathbf{T}(1,1)$ is a symmetric traceless tensor, $A_{\mu \nu}=A_{\mu \nu}^{+}+A_{\mu \nu}^{-} \in$ $\mathbf{T}(1,0) \oplus \mathbf{T}(0,1)$ are the selfdual and the antiselfdual antisymmetric tensors, and $\frac{1}{4} g_{\mu \nu} T_{\rho}^{\rho} \in \mathbf{T}(0,0)$ corresponds to the trivial representation defined through the unit tensor. This decomposition corresponds to the Clebsch-Gordan decomposition of the direct product of two vector representations:

$$
\left(\frac{1}{2}, \frac{1}{2}\right) \otimes\left(\frac{1}{2}, \frac{1}{2}\right)=(1,1) \oplus((1,0) \oplus(0,1)) \oplus(0,0)
$$

Now we consider the decomposition of the space of tensors of rank $n$ into irreducible representation spaces of $S O(1,3 ; \mathbb{R})$. The different symmetry classes are strongly restricted by the requirement that only such Young patterns $[m]$ are allowed for which the sum of the first two colums is lower or equal to four. Therefore, only the following Young patterns correspond to nontrivial irreducible representations by traceless tensors: 


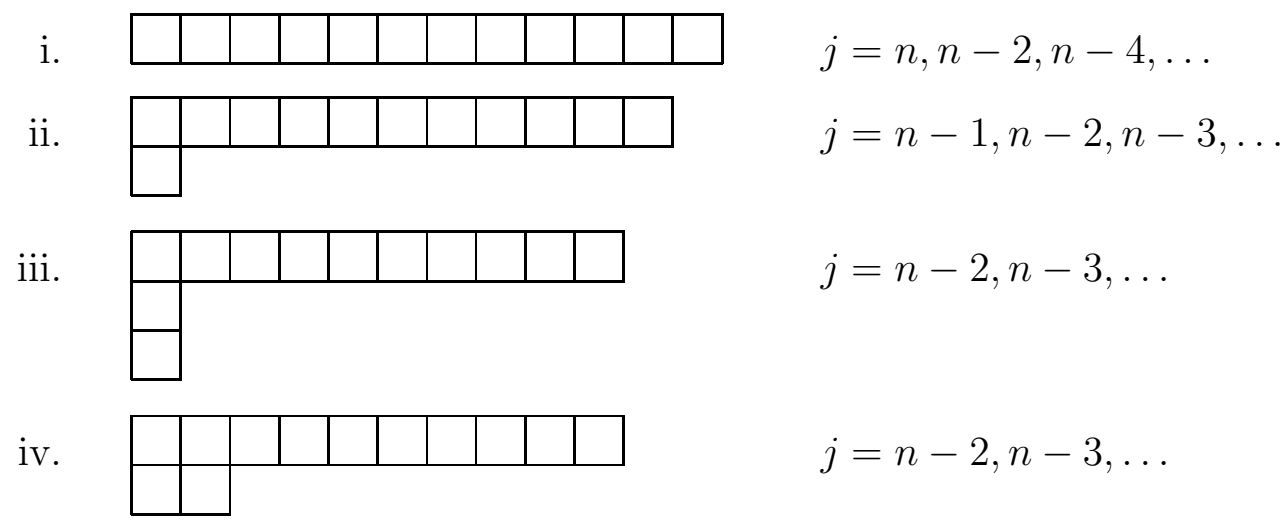

In addition, for $n=4$, also the completely antisymmetric tensor of rank 4 which is proportional to $\epsilon_{\mu \nu \kappa \lambda}$, and therefore equivalent to the trivial representation is allowed.

For the cases (i) - (iv) the minimal spin $j$-depending on $n$ being either even or odd - will be zero or one. In the case of symmetry type (iv) we have given only one special Young pattern; in principle the lenght of the second row may contain up to $m_{2}=\left[\frac{n}{2}\right]$ boxes, and then the maximal spin is given by $j=n-m_{2}$. - The representations corresponding to symmetry class (i) are associated to representations of the symmetry class (iii) with $n+2$ boxes. The representations corresponding to symmetry class (ii) and (iv) are selfassociated. The symmetry class (ii) contains two non-equivalent parts being related to $\left(\frac{n}{2}, \frac{n}{2}-1\right)$ and $\left(\frac{n}{2}-1, \frac{n}{2}\right)$; the symmetry class (iv) contains three nonequivalent parts related to $\left(\frac{n}{2}, \frac{n}{2}-2\right)$, $\left(\frac{n}{2}-1, \frac{n}{2}-1\right)$ and $\left(\frac{n}{2}-2, \frac{n}{2}\right)$; and so on. Any tensor whose symmetry class does not coincide with one of the above classes vanishes identically due to the requirement of tracelessness.

There are two possible ways to construct the nonvanishing tensors. Either one symmetrizes the indices according to the corresponding (standard) Young tableaux and afterwards subtracts the traces, or one starts from tensors being already traceless and finally symmetrizes because this does not destroy the tracelessness. For practical reasons the latter procedure seems to be preferable and will be used in the construction of irreducible light-cone operators of definite twist.

\section{Twist decomposition of nonlocal LC operators}

This Chapter is devoted to show how the LC operators may be decomposed according to their twist being defined by the rule twist $(\tau)=\operatorname{dimension}(d)-\operatorname{spin}(j)$. The procedure to be used is the following:

- first, for arbitrary values of $x$ we expand the nonlocal operators into a Taylor series of local tensor operators having definite rank and mass dimension,

- second, we decompose these tensor operators with respect to irreducible representations of the Lorentz group or, equivalently, the group $S O(4)$ having definite spin and therefore also well-defined twist, 
- third, we resum the infinite series of irreducible tensor operators of equal twist (for any $n$ ) to a nonlocal harmonic operator of definite twist which contains, through its trace terms, also infinitely many operators of higher twist related to it, and

- finally, we project onto the light-cone, $x \rightarrow \tilde{x}$, to obtain the required twist decomposition of the original light-cone operators.

The nonlocal quark operators to be considered and their Taylor expansions are given by:

$$
\begin{aligned}
O^{\Gamma}\left(\kappa_{1} \tilde{x}, \kappa_{2} \tilde{x}\right) & =\bar{\psi}\left(\kappa_{1} \tilde{x}\right) \Gamma U\left(\kappa_{1} \tilde{x}, \kappa_{2} \tilde{x}\right) \psi\left(\kappa_{2} \tilde{x}\right) \\
& =\lim _{x \rightarrow \tilde{x}} \sum_{n=0}^{\infty} \frac{1}{n !} x^{\mu_{1}} \ldots x^{\mu_{n}} \bar{\psi}(0) \Gamma \mathrm{D}_{\mu_{1}}\left(\kappa_{1}, \kappa_{2}\right) \ldots \mathrm{D}_{\mu_{n}}\left(\kappa_{1}, \kappa_{2}\right) \psi(0),
\end{aligned}
$$

where the phase factor $U\left(\kappa_{1} \tilde{x}, \kappa_{2} \tilde{x}\right)$ has been defined by eq. (1.5) and

$$
\Gamma=\left\{1, \gamma_{\mu}, \sigma_{\mu \nu}, \gamma_{5} \gamma_{\mu}, \gamma_{5}\right\} \quad \text { with } \quad \sigma_{\mu \nu}=\frac{i}{2}\left[\gamma_{\mu}, \gamma_{\nu}\right]=\frac{i}{2} \epsilon_{\mu \nu}{ }^{\kappa \lambda} \gamma_{5} \sigma_{\kappa \lambda}
$$

determines a specific $\gamma$-structure (and, if necessary, also the flavour content) of the operators under considerationf; furthermore we have introduced the notation

$$
\begin{gathered}
\mathrm{D}_{\mu}\left(\kappa_{1}, \kappa_{2}\right) \equiv\left(\kappa_{1} \overleftarrow{D}+\kappa_{2} \vec{D}\right)_{\mu} \\
\text { with } \quad \vec{D}_{\mu} \equiv D_{\mu}(A)=\partial_{\mu}+\mathrm{i} g A_{\mu}(x) \text { and } \overleftarrow{D}_{\mu}=\overleftarrow{\partial}_{\mu}-\mathrm{i} g A_{\mu}(x)
\end{gathered}
$$

Obviously, the expansion (3.2) of $O^{\Gamma}\left(\kappa_{1}, \kappa_{2}\right)$ into a Taylor series makes use of the translation of the field operators, $\psi(\kappa x)=\exp (\mathrm{i} \kappa x P) \psi(0) \exp (-\mathrm{i} \kappa x P)$, together with $\left[P_{\mu}, \psi(0)\right]=-\mathrm{i}\left(\partial_{\mu} \psi\right)(0)$.

The local operators appearing in eqs. (3.2) have a tensor structure which, in general, is reducible with respect to the (orthochronous) Lorentz group. However, since only the tensor structure of these operators is of interest, we may restrict the following considerations to the much simpler case $\left(\kappa_{1}=0, \kappa_{2}=\kappa\right)$ where $\mathrm{D}_{\mu}\left(\kappa_{1}, \kappa_{2}\right)$ reduces to the usual covariant derivative $D_{\mu}$ multiplied by $\kappa$. Any (algebraic) operation which will be used henceforth is unchanged by the replacement $\kappa D_{\mu} \rightarrow \mathrm{D}_{\mu}\left(\kappa_{1}, \kappa_{2}\right)$. This is independent from the fact that the product $\mathrm{D}_{\mu_{1}}\left(\kappa_{1}, \kappa_{2}\right) \ldots \mathrm{D}_{\mu_{n}}\left(\kappa_{1}, \kappa_{2}\right)$, if expanded according to (3.3), decays into a sum of tensors - although different - being equivalent realizations of the same symmetry types. Therefore, the twist decomposition which will be obtained for $O^{\Gamma}(0, \kappa)$ immediately holds with obvious replacements for $O^{\Gamma}\left(\kappa_{1}, \kappa_{2}\right)$.

There is an additional property of the considered operators. Since the product of the covariant derivatives is multiplied by the symmetric tensor $x^{\mu_{1}} \ldots x^{\mu_{n}}$

${ }^{5}$ The symmetry with respect to the exchange of $\kappa_{1}, \kappa_{2}$ and the renormalization procedure of the LC operators have not been explicitly indicated since they do not play any role in our consideration here; for corresponding details see [8]. 
only its symmetric part $D_{\left\{\mu_{1}\right.} \ldots D_{\left.\mu_{n}\right\}}$ is of relevance where the symbol $\{\ldots\}$ denotes symmetrization with respect to the enclosed indices (including division by $n !)$; in the same manner [...] will be used to denote antisymmetrization. Of course, $D_{\left\{\mu_{1}\right.} \ldots D_{\left.\mu_{n}\right\}}$ as any symmetric tensor decomposes into irreducible tensors according to the Clebsch-Gordan series

$$
D_{\left\{\mu_{1}\right.} \ldots D_{\left.\mu_{n}\right\}} \in \mathbf{T}\left(\frac{n}{2}, \frac{n}{2}\right) \oplus \mathbf{T}\left(\frac{n-2}{2}, \frac{n-2}{2}\right) \oplus \mathbf{T}\left(\frac{n-4}{2}, \frac{n-4}{2}\right) \oplus \ldots
$$

where the traceless tensor of order $n$ transform according to $\mathbf{T}\left(\frac{n}{2}, \frac{n}{2}\right)$, and any of the further representations correspond to the traces which contain an increasing number of metric tensors (multiplied by $x^{2}$ ). Therefore, in the limit $x \rightarrow \tilde{x}$ only the tensor space $\mathbf{T}\left(\frac{n}{2}, \frac{n}{2}\right)$ contributes. This leads to essential restrictions for the decomposition of the LC-operators under consideration.

The indices resulting from the Taylor decomposition of the LC-operators will be called "internal" ones, whereas the indices which are due to the nonlocal operator itself, i.e., which are related to $\Gamma$ will be called "external". For some physical situations it will be useful to multiply also (some of the) external indices by $x^{\mu}$ and $\tilde{x}^{\mu}$, respectively, thereby extending the above conclusion.

\section{$3.1 \quad$ (Pseudo) Scalar operators}

The simplest cases to demonstrate how the method works are the (pseudo) scalar operators. In order to think of some specific example we could take $\Gamma=\{1,(\tilde{x} \gamma)=$ $\left.\tilde{x}^{\mu} \gamma_{\mu},(\tilde{x} \sigma \partial)=\tilde{x}^{\mu} \sigma_{\mu \nu} \partial^{\nu}, \gamma_{5}(\tilde{x} \gamma), \gamma_{5}\right\}$ leading to operators having lowest twist $\tau_{\min }=$ $\{3,2,2,2,3\}$, respectively. Here, any Lorentz index of the local operators is an internal one and only the Young tableaux of type (i) - corresponding to complete symmetrisation - are of relevance. According to eq. (3.5) they contain contributions of twist $\tau_{n}=\tau_{\min }+2 n, n=0,1,2, \ldots$ which, on the light-cone, reduce to $\tau_{\min }$ only.

To be definite, let us consider the operator

$$
O(0, \kappa x)=\bar{\psi}(0) x U(0, \kappa x) \psi(\kappa x),
$$

which also appears in the consideration of vector operators. Its decomposition into local tensor operators reads

$$
\sum_{n=0}^{\infty} \frac{\kappa^{n}}{n !} x^{\beta} x^{\mu_{1}} \ldots x^{\mu_{n}} \bar{\psi}(0) \gamma_{\beta} D_{\mu_{1}} \ldots D_{\mu_{n}} \psi(0)=\sum_{n=0}^{\infty} \frac{\kappa^{n}}{n !} \bar{\psi}(0) \not(x D)^{n} \psi(0)
$$

with the obvious abbreviation $(x D)^{n} \equiv x^{\mu_{1}} \ldots x^{\mu_{n}} D_{\mu_{1}} \ldots D_{\mu_{n}}$.

Now, the local operators $\bar{\psi}(0) \gamma_{\beta} D_{\mu_{1}} \ldots D_{\mu_{n}} \psi(0)$ have to be made traceless. This is easily achieved if we observe that a totally symmetric traceless tensor $\stackrel{\circ}{T}_{\mu_{1} \ldots \mu_{n}}$ whose indices are completely saturated by contracting with $x^{\mu_{1}} \ldots x^{\mu_{n}}$ is 
a harmonic polynomial of degree $n$. It obeys the 4-dimensional Laplace equation if continued to Euclidean space-time,

$$
\square \stackrel{\circ}{T}_{n}(x)=0 \quad \text { with } \quad \stackrel{\circ}{T}_{n}(x):=x^{\mu_{1}} \ldots x^{\mu_{n}} \stackrel{\circ}{T}_{\mu_{1} \ldots \mu_{n}}
$$

which is obvious if one observes that $\square x^{\mu} x^{\nu} \equiv 2 g^{\mu \nu}$. The solution of eq. (3.8) is given by (see Appendix B, eq. (B.5)):

$$
\stackrel{\circ}{T}_{n}(x)=\sum_{k=0}^{\left[\frac{n}{2}\right]} \frac{(n-k) !}{n ! k !}\left(\frac{-x^{2}}{4}\right)^{k} \square^{k} x^{\mu_{1}} \ldots x^{\mu_{n}} T_{\mu_{1} \ldots \mu_{n}} \equiv H_{n}^{(4)}\left(x^{2} \mid \square\right) T_{n}(x) .
$$

From this we conclude, after replacing $n \rightarrow n+1$, that the local traceless quark operators in eq. (3.7) read

$$
\left[\bar{\psi}(0) \not x(x D)^{n} \psi(0)\right]^{\text {traceless }}=\sum_{k=0}^{\left[\frac{n+1}{2}\right]} \frac{(n+1-k) !}{(n+1) ! k !}\left(\frac{-x^{2}}{4}\right)^{k} \square^{k} \bar{\psi}(0) \not x(x D)^{n} \psi(0) .
$$

Let us remark that the summation over $k$ could be left unbounded since, due to the $k$-th power of the Laplacian, it automatically terminates at $\left[\frac{n+1}{2}\right]$.

Now, these traceless operators of twist- 2 must be resummed by introducing them into eq. (3.7). Using the integral representation of Euler's beta function,

$$
B(n, m)=\frac{\Gamma(n) \Gamma(m)}{\Gamma(n+m)}=\int_{0}^{1} \mathrm{~d} t t^{n-1}(1-t)^{m-1},
$$

in order to replace $(n+1-k) ! /(n+1) !=B(n+2-k, k) /(k-1)$ ! we obtain the following traceless operator

$$
\sum_{n=0}^{\infty}\left[1+\sum_{k=1}^{\infty} \int_{0}^{1} \mathrm{~d} t\left(\frac{-x^{2}}{4}\right)^{k} \frac{\square^{k} t^{n}}{k !(k-1) !}\left(\frac{1-t}{t}\right)^{k-1}\right] \frac{\kappa^{n}}{n !} \bar{\psi}(0) \not(x D)^{n} \psi(0) .
$$

Here, the sum over $n$ can be carried out leading to

$$
\begin{aligned}
\stackrel{\circ}{O}(0, \kappa x)= & \bar{\psi}(0) \not x U(0, \kappa x) \psi(\kappa x) \\
& +\sum_{k=1}^{\infty} \int_{0}^{1} \mathrm{~d} t\left(\frac{-x^{2}}{4}\right)^{k} \frac{\square^{k}}{k !(k-1) !}\left(\frac{1-t}{t}\right)^{k-1} \bar{\psi}(0) \not U(0, \kappa t x) \psi(\kappa t x) .
\end{aligned}
$$

This expression already has been dealt with by [4] without mentioning its derivation. It is obvious that the terms corresponding to $k=1,2, \ldots$ contain operators of higher twist $\tau=4,6, \ldots$ which, because they are multiplied with $x^{2}$, do not contribute on the light-cone. Therefore, the scalar light-cone operator $\bar{\psi}(0) \gamma \tilde{x} U(0, \kappa \tilde{x}) \psi(\kappa \tilde{x})$ is already of twist-2. 
Let us observe that the derivation of (3.11) is independent of the origin of the specific structure of the local tensors appearing in eq. (3.7). Therefore, we may replace $\kappa D_{\mu}$ by $\mathrm{D}_{\mu}\left(\kappa_{1}, \kappa_{2}\right)$ without changing the final result:

$$
\begin{aligned}
& \stackrel{\circ}{O}\left(\kappa_{1} x, \kappa_{2} x\right)=\bar{\psi}\left(\kappa_{1} x\right) \not U\left(\kappa_{1} x, \kappa_{2} x\right) \psi\left(\kappa_{2} x\right) \\
& \quad+\sum_{k=1}^{\infty} \int_{0}^{1} \mathrm{~d} t\left(\frac{-x^{2}}{4}\right)^{k} \frac{\square^{k}}{k !(k-1) !}\left(\frac{1-t}{t}\right)^{k-1} \bar{\psi}\left(\kappa_{1} t x\right) \not U\left(\kappa_{1} t x, \kappa_{2} t x\right) \psi\left(\kappa_{2} t x\right) .
\end{aligned}
$$

The projection onto the light-cone leads to

$$
O^{\mathrm{tw} 2}\left(\kappa_{1} \tilde{x}, \kappa_{2} \tilde{x}\right)=\bar{\psi}\left(\kappa_{1} \tilde{x}\right)(\tilde{x} \gamma) U\left(\kappa_{1} \tilde{x}, \kappa_{2} \tilde{x}\right) \psi\left(\kappa_{2} \tilde{x}\right)
$$

of course, any of the scalar structures $\Gamma$ introduced above could be used instead of $(\tilde{x} \gamma)$.

\section{2 (Pseudo) Vector operators}

Let us now consider the (pseudo) vector operators with $\Gamma$ being $\gamma_{\beta}$ (or $\gamma_{\beta} \gamma_{5}$ ):

$$
O_{\beta}(0, \kappa x)=\bar{\psi}(0) \gamma_{\beta} U(0, \kappa x) \psi(\kappa x)
$$

whose local tensor operators differ from those in eq. (3.7) only by the absence of $x^{\beta}$. They are given by

$$
\begin{aligned}
O_{\beta \mu_{1} \ldots \mu_{n}} & \equiv \bar{\psi}(0) \gamma_{\beta} D_{\left\{\mu_{1}\right.} \ldots D_{\left.\mu_{n}\right\}} \psi(0) \\
& =\bar{\psi}(0) \gamma_{\{\beta} D_{\mu_{1}} \ldots D_{\left.\mu_{n}\right\}} \psi(0)+\alpha_{n} \bar{\psi}(0) \gamma_{[\beta} D_{\left\{\mu_{1}\right]} \ldots D_{\left.\mu_{n}\right\}} \psi(0)+\ldots
\end{aligned}
$$

they decompose into two parts being related to the Young patterns (i) and (ii) as well as to additional contributions being antisymmetric in (some of) the $\mu$ 's, and $\alpha_{n}=2 n /(n+1)$ results from the nontrivial normalization of the Young operator $\mathcal{Y}_{[m]}$ with $[m]=(n, 1)$ (see Appendix A, eqs. (A.4) and (A.7)). Let us remind that because of the full reducibility of the direct product of a vector with a completely symmetric tensor (3.15) corresponds to the following Clebsch-Gordan series

$$
\begin{aligned}
\left(\frac{1}{2},\right. & \left.\frac{1}{2}\right) \otimes\left(\left(\frac{n}{2}, \frac{n}{2}\right) \oplus\left(\frac{n-2}{2}, \frac{n-2}{2}\right) \oplus \ldots\right) \\
=\left(\frac{n+1}{2}, \frac{n+1}{2}\right) & \oplus\left(\left(\frac{n+1}{2}, \frac{n-1}{2}\right) \oplus\left(\frac{n-1}{2}, \frac{n+1}{2}\right)\right) \oplus 2\left(\frac{n-1}{2}, \frac{n-1}{2}\right) \oplus \ldots .
\end{aligned}
$$

The mass dimension of the operator (3.14) is $n+3$, and the spin of the various contributions in (3.17) ranges from $n+1$ up to 1 or 0 if $n$ is even or odd, respectively; therefore the twist decomposition reads:

$$
O_{\beta \mu_{1} \ldots \mu_{n}}=O_{\beta \mu_{1} \ldots \mu_{n}}^{\mathrm{tw} 2}+O_{\beta \mu_{1} \ldots \mu_{n}}^{\mathrm{tw} 3}+O_{\beta \mu_{1} \ldots \mu_{n}}^{\mathrm{tw} 4}+\ldots
$$

with the last term in the series being $O_{\beta \mu_{1} \ldots \mu_{n}}^{\mathrm{tw}(\mathrm{n}+2)}$ or $O_{\beta \mu_{1} \ldots \mu_{n}}^{\mathrm{tw}(\mathrm{n}+3)}$, respectively. From this it follows that the operator (3.2) for arbitrary $x$ contains contributions of any 
twist, $\tau \geq 2$. However, in the limit $x \rightarrow \tilde{x}$ only the first three terms of eq. (3.18) survive since the contributions for $\tau>4$ are proportional to $x^{2}$. Therefore we obtain the following decomposition:

$$
O_{\beta}(0, \kappa \tilde{x})=O_{\beta}^{\mathrm{tw} 2}(0, \kappa \tilde{x})+O_{\beta}^{\mathrm{tw} 3}(0, \kappa \tilde{x})+O_{\beta}^{\mathrm{tw} 4}(0, \kappa \tilde{x}) .
$$

On the light-cone the totally symmetric part of eq. (3.16) contributes to $\tau=2,4$, and the partially antisymmetric part of (3.16) contributes to $\tau=3,4$. Obviously, the twist-4 operator consists of two independent parts.

(A) Totally symmetric vector operators:

The totally symmetric traceless tensors which have twist $\tau=2$ are contained in $\mathbf{T}\left(\frac{n+1}{2}, \frac{n+1}{2}\right)$ and they are characterized by the following standard tableaux:

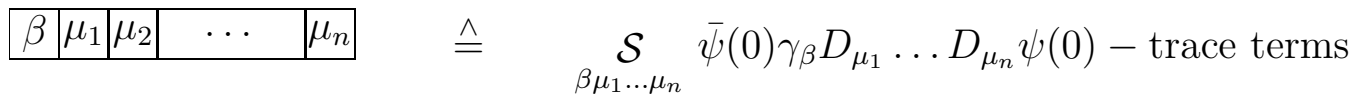

where the normalizing factor is 1 . Let us write this operator in the form

$$
\begin{aligned}
O_{\beta \mu_{1} \ldots \mu_{n}}^{\mathrm{tw} 2}= & \frac{1}{n+1}\left(\bar{\psi}(0) \gamma_{\beta} D_{\left\{\mu_{1}\right.} \ldots D_{\left.\mu_{n}\right\}} \psi(0)\right. \\
& \left.+\sum_{l=1}^{n} \bar{\psi}(0) \gamma_{\left\{\mu_{l}\right.} D_{\mu_{1}} \ldots D_{\mu_{l-1}} D_{|\beta|} D_{\mu_{l+1}} \ldots D_{\left.\mu_{n}\right\}} \psi(0)\right) \text {-trace terms }
\end{aligned}
$$

where the symbol $|\ldots|$ indicates that the enclosed indices are to be excluded from the symmetrization caused by $\{\ldots\}$. If the trace terms in (3.20) had been determined, this tensor would be irreducible. Let us postpone that determination and make the resummation to the corresponding nonlocal operator in advance. This is obtained by contracting with $x^{\mu_{1}} \ldots x^{\mu_{n}}$ :

$$
O_{\beta n}^{\mathrm{tw} 2}(x)=x^{\mu_{1}} \ldots x^{\mu_{n}} O_{\beta \mu_{1} \ldots \mu_{n}}^{\mathrm{tw} 2}=\frac{1}{n+1} \partial_{\beta}\left(\bar{\psi}(0) \not \partial(x D)^{n} \psi(0)-\text { trace terms }\right) .
$$

The trace terms of $\bar{\psi}(0) \not x(x D)^{n} \psi(0)$ have been determined already in Subsection 3.1. Therefore, using $(n+1)^{-1}=\int_{0}^{1} d \lambda \lambda^{n}$ we obtain:

$$
O_{\beta}^{\mathrm{tw} 2}(0, \kappa x)=\sum_{n=0}^{\infty} \frac{\kappa^{n}}{n !} O_{\beta n}^{\mathrm{tw} 2}(x)=\partial_{\beta} \int_{0}^{1} \mathrm{~d} \lambda \stackrel{\circ}{\circ}(0, \kappa \lambda x),
$$

with $\stackrel{\circ}{O}(0, \kappa \lambda x)$ being defined through eq. (3.11). Of course, the derivative $\partial_{\beta}$ could be taken outside the parentheses in eq. (3.21) (and the integral) because the Young operators should be applied to traceless tensors. (For notational definiteness we remark that partial derivatives are everywhere with respect to $x$ only.) Note that $x^{\beta} O_{\beta}^{\text {tw2 }}(0, \kappa x)=\stackrel{\circ}{O}(0, \kappa x)$ is obtained from eq. (3.22) by partial integration observing that for any function $f(\lambda x)$ the equality $x^{\mu} \partial f / \partial x^{\mu}=\lambda \partial f / \partial \lambda$ holds. Analogous to the scalar case the generalization to arbitrary values $\left(\kappa_{1}, \kappa_{2}\right)$ is

$$
O_{\beta}^{\mathrm{tw} 2}\left(\kappa_{1} x, \kappa_{2} x\right)=\partial_{\beta} \int_{0}^{1} \mathrm{~d} \lambda \stackrel{\circ}{O}\left(\kappa_{1} \lambda x, \kappa_{2} \lambda x\right),
$$


with $\stackrel{\circ}{O}\left(\kappa_{1} \lambda x, \kappa_{2} \lambda x\right)$ defined through eq. (3.12). By construction this traceless harmonic vector operator fulfills the equations

$$
\square O_{\beta}^{\mathrm{tw} 2}\left(\kappa_{1} x, \kappa_{2} x\right)=0, \quad \partial^{\beta} O_{\beta}^{\mathrm{tw} 2}\left(\kappa_{1} x, \kappa_{2} x\right)=0 .
$$

Let us now project onto the light-cone. Since only one differentiation $\partial_{\beta}$ appears in eq. (3.22) and (3.23) the whole sum in (3.11) and (3.12), respectively, contributes on the light-cone only through the term $k=1$. Using the relation

$$
\int_{0}^{1} \mathrm{~d} \lambda \int_{0}^{1} \mathrm{~d} t f(\kappa \lambda t)=-\int_{0}^{1} \mathrm{~d} \lambda(\ln \lambda) f(\kappa \lambda)
$$

we finally obtain for the nonlocal twist-2 light-cone operator

$$
\begin{aligned}
O_{\beta}^{\mathrm{tw} 2}\left(\kappa_{1} \tilde{x}, \kappa_{2} \tilde{x}\right) & =\lim _{x \rightarrow \tilde{x}} \partial_{\beta} \int_{0}^{1} \mathrm{~d} \lambda\left[1+\frac{\ln \lambda}{4} x^{2} \square\right] \bar{\psi}\left(\kappa_{1} \lambda x\right) \not x U\left(\kappa_{1} \lambda x, \kappa_{2} \lambda x\right) \psi\left(\kappa_{2} \lambda x\right) \\
& =\left.\int_{0}^{1} \mathrm{~d} \lambda\left[\partial_{\beta}+\frac{\ln \lambda}{2} x_{\beta} \square\right] \bar{\psi}\left(\kappa_{1} \lambda x\right) \not x U\left(\kappa_{1} \lambda x, \kappa_{2} \lambda x\right) \psi\left(\kappa_{2} \lambda x\right)\right|_{x=\tilde{x}} .
\end{aligned}
$$

Obviously, the second term of the integrand which is proportional to $x_{\beta}$, and which results from $k=1$, is the trace term; up to an overall minus sign it is the (symmetric) twist -4 vector operator

$$
O_{\beta, \mathrm{sym}}^{\mathrm{tw} 4}\left(\kappa_{1} \tilde{x}, \kappa_{2} \tilde{x}\right)=-\left.\frac{1}{2} \tilde{x}_{\beta} \square \int_{0}^{1} \mathrm{~d} \lambda \ln \lambda \bar{\psi}\left(\kappa_{1} \lambda x\right) \not x U\left(\kappa_{1} \lambda x, \kappa_{2} \lambda x\right) \psi\left(\kappa_{2} \lambda x\right)\right|_{x=\tilde{x}} .
$$

Of course, if we multiply (3.26) with $\tilde{x}^{\beta}$ the twist-4 part vanishes and the remaining contributions restore the scalar operator (3.13).

Furthermore, after performing the differentiations in (3.26) we can use the axial gauge, $\tilde{x}^{\mu} A_{\mu}=0$, in order to avoid the phase factor, $U\left(\kappa_{1} \tilde{x}, \kappa_{2} \tilde{x}\right)=1$; then we obtain

$O_{\beta}^{\mathrm{tw} 2}\left(\kappa_{1} \tilde{x}, \kappa_{2} \tilde{x}\right)=\left.\int_{0}^{1} \mathrm{~d} \lambda \bar{\psi}\left(\kappa_{1} y\right)\left(\gamma_{\beta}+y \mathrm{D}_{\beta}+y_{\beta} \ln \lambda\left[\not D+\frac{1}{2} y \mathrm{D}^{2}\right]\right) \psi\left(\kappa_{2} y\right)\right|_{y=\lambda \tilde{x}}$,

with the convention that the covariant left-right derivative in axial gauge,

$$
\mathrm{D}_{\beta} \equiv \overleftarrow{\partial_{\beta}^{y}}-i g \int_{\kappa_{2}}^{\kappa_{1}} d \tau A_{\beta}(\tau y)+\overrightarrow{\partial_{\beta}^{y}},
$$

acts only on the arguments of the quark and gluon fields. (This convention which is used also in the following, is necessary only to simplify the notation.)

Finally, it should be remarked, that the conditions (3.24), if translated into the corresponding ones with respect to $\tilde{x}$, no longer hold for the light-cone operators (3.26). This is obvious because by projecting onto the light-cone part of the original structure of the operators has been lost. Nevertheless, the resulting lightcone operators are restrictions of harmonic functions. This was the reason why 
in order to determine the correct operators of definite twist we started with the quark operators for arbitrary values of $x$.

(B) Vector operators having symmetry class (ii)

Now we consider the symmetry class (ii) which contributes to spin $j=n$ and $n-1$. Because of the specific symmetry of the local operators (3.15) there appears only one nontrivial standard tableaux:?

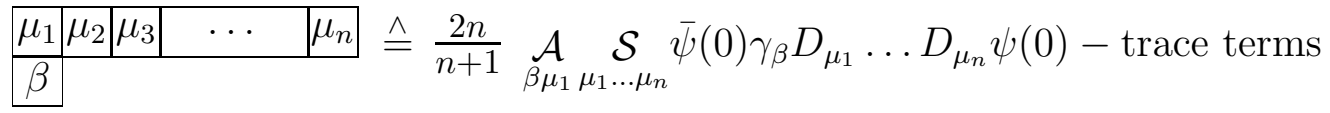

with the normalization $\alpha_{n}=2 n /(n+1)$ of the Young operator. The corresponding traceless tensor being contained in $\mathbf{T}\left(\frac{n+1}{2}, \frac{n-1}{2}\right) \oplus \mathbf{T}\left(\frac{n-1}{2}, \frac{n+1}{2}\right)$ has twist $\tau=3$; it is reducible with respect to $S O(1,3)$, but irreducible with respect to $O(1,3)$ :

$$
O_{\beta \mu_{1} \ldots \mu_{n}}^{\mathrm{tw} 3}=\frac{n}{n+1} \bar{\psi}(0)\left(\gamma_{\beta} D_{\left\{\mu_{1}\right.} \ldots D_{\left.\mu_{n}\right\}}-\gamma_{\mu_{1}} D_{\{\beta} \ldots D_{\left.\mu_{n}\right\}}\right) \psi(0)-\text { trace terms. }
$$

This irreducible tensor will be contracted with $x^{\mu_{1}} \ldots x^{\mu_{n}}$ to obtain:

$$
O_{\beta n}^{\mathrm{tw} 3}(x)=\frac{2}{n+1} \frac{1}{2}\left(g_{\alpha \beta}(x \partial)-x_{\alpha} \partial_{\beta}\right) \bar{\psi}(0) \gamma^{\alpha}(x D)^{n} \psi(0)-\text { trace terms } .
$$

Obviously, the differential operator $\frac{1}{2}\left(g_{\beta \alpha}(x \partial)-x_{\alpha} \partial_{\beta}\right)$ takes care of the antisymmetrization $\mathcal{A}_{\beta \mu_{i}}$ after having symmetrized by contracting with the $x$ 's. However, this contraction in addition causes that those contributions to (3.16) which are antisymmetric in some of the $\mu$ 's vanish such that the following relation holds

$$
x^{\mu_{1}} \ldots x^{\mu_{n}} O_{\beta \mu_{1} \ldots \mu_{n}}=x^{\mu_{1}} \ldots x^{\mu_{n}}\left(\underset{\beta \mu_{1} \ldots \mu_{n}}{\mathcal{S}} O_{\beta \mu_{1} \ldots \mu_{n}}+\frac{2 n}{n+1} \underset{\beta \mu_{1} \mu_{1} \ldots \mu_{n}}{\mathcal{S}} O_{\beta \mu_{1} \ldots \mu_{n}}\right) .
$$

Of course, this relation could have been used to determine the prefactor $\alpha_{n}$ without any knowledge of the normalization of the Young operators.1

Again, before determining the trace terms we resum these irreducible local tensors to the corresponding nonlocal operators:

$$
O_{\beta}^{\mathrm{tw} 3}(0, \kappa x)=\left(\delta_{\beta}^{\alpha}(x \partial)-x^{\alpha} \partial_{\beta}\right) \int_{0}^{1} \mathrm{~d} \lambda\left(O_{\alpha}(0, \kappa \lambda x)-\text { trace terms }\right) .
$$

In distinction to eq. (3.8) we now have to determine a traceless tensor with a free index $\alpha, \stackrel{\circ}{T}_{\alpha n}(x) \equiv x^{\mu_{1}} \ldots x^{\mu_{n}} \stackrel{\circ}{T}_{\alpha \mu_{1} \ldots \mu_{n}}$, obeying the following two equations:

$$
\square \stackrel{\circ}{T}_{\alpha n}(x)=0 \quad \text { and } \quad \partial^{\alpha} \stackrel{\circ}{T}_{\alpha n}(x)=0,
$$

${ }^{6}$ Local operators with this symmetry have been considered first by [1], 23] in connection with the polarized deep inelastic scattering.

${ }^{7}$ In fact, contraction with the $x$ 's could be understood as a supplementary symmetrization according to $\mathcal{S}_{\mu_{1} \ldots \mu_{n}} \mathcal{A}_{\beta \mu_{1}} \mathcal{S}_{\mu_{1} \ldots \mu_{n}}$ which has been first considered by [31, 13]. It corresponds to taking into account all the $n$ ! equivalent representations related to the above Young pattern or simply replacing $\gamma_{\mu_{1}} D_{\{\beta} \ldots D_{\left.\mu_{n}\right\}}$ by $\frac{1}{n} \sum_{\ell} \gamma_{\mu_{\ell}} D_{\left\{\mu_{1}\right.} \ldots D_{\mu_{\ell-1}} D_{\beta} D_{\mu_{\ell+1}} \ldots D_{\left.\mu_{n}\right\}}$. Obviously, this additional symmetry operation destroys the irreducibility and, furthermore, applied to tensors being not traceless it may change the structure of the trace terms. 
which correspond to taking the traces $g_{\mu_{i} \mu_{j}}$ and $g_{\alpha \mu_{i}}$, respectively. The solution of these equations reads (see Appendix B, eq. (B.9)):

$$
\stackrel{\circ}{T}_{\alpha n}=\left\{\delta_{\alpha}^{\mu}-\frac{1}{(n+1)^{2}}\left[x_{\alpha} \partial^{\mu}(x \partial)-\frac{1}{2} x^{2} \partial_{\alpha} \partial^{\mu}\right]\right\} \sum_{k=0}^{\left[\frac{n}{2}\right]} \frac{(n-k) !}{k ! n !}\left(\frac{-x^{2}}{4}\right)^{k} \square^{k} T_{\mu n}(x) .
$$

Again, using (3.10) and the integral representation of $1 /(n+1)$ we obtain

$$
\begin{aligned}
& \stackrel{\circ}{O}_{\alpha}(0, \kappa x)=O_{\alpha}(0, \kappa x)+\sum_{k=1}^{\infty} \int_{0}^{1} \frac{\mathrm{d} t}{t}\left(\frac{-x^{2}}{4}\right)^{k} \frac{\square^{k}}{k !(k-1) !}\left(\frac{1-t}{t}\right)^{k-1} O_{\alpha}(0, \kappa t x) \\
& \quad-\left[x_{\alpha} \partial^{\mu}(x \partial)-\frac{1}{2} x^{2} \partial_{\alpha} \partial^{\mu}\right] \sum_{k=0}^{\infty} \int_{0}^{1} \mathrm{~d} \tau \int_{0}^{1} \mathrm{~d} t\left(\frac{-x^{2}}{4}\right)^{k} \frac{\square^{k}}{k ! k !}\left(\frac{1-t}{t}\right)^{k} O_{\mu}(0, \kappa \tau t x) .
\end{aligned}
$$

For the harmonic vector operator of twist $\tau=3$ we obtain:

$$
O_{\beta}^{\mathrm{tw} 3}(0, \kappa x)=\left(\delta_{\beta}^{\alpha}(x \partial)-x^{\alpha} \partial_{\beta}\right) \int_{0}^{1} \mathrm{~d} \lambda \stackrel{\circ}{\circ}_{\alpha}(0, \kappa \lambda x)
$$

and its generalization to arbitrary values of $\left(\kappa_{1}, \kappa_{2}\right)$ simply reads

$$
O_{\beta}^{\mathrm{tw} 3}\left(\kappa_{1} x, \kappa_{2} x\right)=\left(\delta_{\beta}^{\alpha}(x \partial)-x^{\alpha} \partial_{\beta}\right) \int_{0}^{1} \mathrm{~d} \lambda \stackrel{\circ}{\circ}_{\alpha}\left(\kappa_{1} \lambda x, \kappa_{2} \lambda x\right)
$$

it fulfills the equations

$$
\square O_{\beta}^{\mathrm{tw} 3}\left(\kappa_{1} x, \kappa_{2} x\right)=0, \quad \partial^{\beta} O_{\beta}^{\mathrm{tw} 3}\left(\kappa_{1} x, \kappa_{2} x\right)=0 .
$$

Because of the various differential operators which come into the play by formulas (3.35) or (3.36) it is not as easy as in the case of scalar operators to discriminate between the different trace terms.

In the limit $x \rightarrow \tilde{x}$ only the terms with $k=0,1$ and 2 of (3.34) survive. The integrals which appear in these formulas may be rewritten as:

$$
\begin{aligned}
\int_{0}^{1} \mathrm{~d} \lambda \int_{0}^{1} \frac{\mathrm{d} t}{t} f(\kappa \lambda t) & =\int_{0}^{1} \mathrm{~d} \lambda \frac{1-\lambda}{\lambda} f(\kappa \lambda), \\
\int_{0}^{1} \mathrm{~d} \lambda \int_{0}^{1} \mathrm{~d} \tau \int_{0}^{1} \mathrm{~d} t f(\kappa \lambda \tau t) & =\int_{0}^{1} \mathrm{~d} \lambda\left(\frac{1}{2} \ln ^{2} \lambda\right) f(\kappa \lambda), \\
\int_{0}^{1} \mathrm{~d} \lambda \int_{0}^{1} \mathrm{~d} \tau \int_{0}^{1} \mathrm{~d} t \frac{1-t}{t} f(\kappa \lambda \tau t) & =\int_{0}^{1} \mathrm{~d} \lambda\left(\frac{1-\lambda}{\lambda}+\ln \lambda-\frac{1}{2} \ln ^{2} \lambda\right) f(\kappa \lambda) .
\end{aligned}
$$

Then for the nonlocal light-ray operator of twist-3 we get:

$$
\begin{aligned}
& O_{\beta}^{\mathrm{tw} 3}(0, \kappa \tilde{x})=\int_{0}^{1} \mathrm{~d} \lambda\left(g_{\beta \alpha}(x \partial)-x_{\alpha} \partial_{\beta}\right)\left[\left\{1-\frac{1-\lambda}{\lambda} \frac{x^{2}}{4} \square\right\} g^{\alpha \mu}\right. \\
& \left.\quad-\left[x^{\alpha} \partial^{\mu}(x \partial)-\frac{1}{2} x^{2} \partial^{\alpha} \partial^{\mu}\right]\left(\frac{\ln ^{2} \lambda}{2}-\left\{\frac{1-\lambda}{\lambda}+\ln \lambda-\frac{\ln ^{2} \lambda}{2}\right\} \frac{x^{2}}{4} \square\right)\right]\left.O_{\mu}(0, \kappa \lambda x)\right|_{x=\tilde{x}}
\end{aligned}
$$


Now, the various differentiations should be carried out. Again, using $(x \partial) f(\lambda x)$ $=\lambda \partial f(\lambda x) / \partial \lambda$ and performing the partial integrations with respect to $\lambda$, after generalization to arbitrary values of $\kappa_{i}$, we finally obtain the simple expression:

$$
\begin{aligned}
O_{\beta}^{\mathrm{tw} 3}\left(\kappa_{1} \tilde{x}, \kappa_{2} \tilde{x}\right)= & \int_{0}^{1} \mathrm{~d} \lambda\left[\left(\delta_{\beta}^{\alpha}(x \partial)-x^{\alpha} \partial_{\beta}\right)\right. \\
& \left.-x_{\beta}\left\{(\ln \lambda) x^{\alpha} \square+(1+2 \ln \lambda) \partial^{\alpha}\right\}\right]\left.O_{\alpha}\left(\kappa_{1} \lambda x, \kappa_{2} \lambda x\right)\right|_{x=\tilde{x}}
\end{aligned}
$$

In axial gauge we obtain (with the above mentioned conventions)

$$
\begin{aligned}
O_{\beta}^{\mathrm{tw} 3}\left(\kappa_{1} \tilde{x}, \kappa_{2} \tilde{x}\right)=\int_{0}^{1} \mathrm{~d} \lambda \bar{\psi}\left(\kappa_{1} y\right)( & {\left[\gamma_{\beta}(y \mathrm{D})-y \mathrm{D}_{\beta}\right] } \\
& \left.-y_{\beta}\left[(\ln \lambda) y \mathrm{D}^{2}+(1+2 \ln \lambda) \not\right]\right)\left.\psi\left(\kappa_{2} y\right)\right|_{y=\lambda \tilde{x}} .
\end{aligned}
$$

Again, the trace terms of (3.42) being proportional to $x_{\beta}$ determine the (partially) antisymmetric bilocal twist -4 vector operator:

$$
O_{\beta, \text { as }}^{\mathrm{tw} 4}\left(\kappa_{1} \tilde{x}, \kappa_{2} \tilde{x}\right)=\left.\tilde{x}_{\beta} \int_{0}^{1} \mathrm{~d} \lambda\left\{(\ln \lambda) x^{\alpha} \square+(1+2 \ln \lambda) \partial^{\alpha}\right\} O_{\alpha}\left(\kappa_{1} \lambda x, \kappa_{2} \lambda x\right)\right|_{x=\tilde{x}} .
$$

Now, having determined the nonlocal operators of twist 2 and 3 we can use either eq. (3.19) to get the nonlocal light-cone operator of twist $\tau=4$ which includes the contributions of the Young patterns (i) and (ii) with spin $n-1$ being contained in $\mathbf{T}\left(\frac{n-1}{2}, \frac{n-1}{2}\right)$, or simply add both twist -4 contributions:

$$
\begin{aligned}
O_{\beta}^{\mathrm{tw} 4}\left(\kappa_{1} \tilde{x}, \kappa_{2} \tilde{x}\right) & =O_{\beta, \mathrm{sym}}^{\mathrm{tw} 4}\left(\kappa_{1} \tilde{x}, \kappa_{2} \tilde{x}\right)+O_{\beta, \text { as }}^{\mathrm{tw} 4}\left(\kappa_{1} \tilde{x}, \kappa_{2} \tilde{x}\right) \\
& =\left.\tilde{x}_{\beta} \int_{0}^{1} \mathrm{~d} \lambda\left[(1+\ln \lambda) \partial^{\alpha}+\frac{\ln \lambda}{2} x^{\alpha} \square\right] O_{\alpha}\left(\kappa_{1} \lambda x, \kappa_{2} \lambda x\right)\right|_{x=\tilde{x}} ;
\end{aligned}
$$

and for the axial gauge we may write

$$
O_{\beta}^{\mathrm{tw} 4}\left(\kappa_{1} \tilde{x}, \kappa_{2} \tilde{x}\right)=\left.\tilde{x}_{\beta} \int_{0}^{1} \mathrm{~d} \lambda \lambda \bar{\psi}\left(\kappa_{1} y\right)\left((1+\ln \lambda) \not D+\frac{\ln \lambda}{2} y \mathrm{D}^{2}\right) \psi\left(\kappa_{2} y\right)\right|_{y=\lambda \tilde{x}}
$$

The corresponding local twist-4 operators read:

$$
O_{\beta n}^{\mathrm{tw} 4}=\left.\frac{\tilde{x}_{\beta}}{2(n+1)^{2}}\left(\partial^{\alpha}(x \partial)-x^{\alpha} \square\right) \bar{\psi}(0) \gamma_{\alpha}(x D)^{n} \psi(0)\right|_{x=\tilde{x}} .
$$

This finishes the decomposition of the nonlocal vector quark operators into its components of definite twist. Quite analogous results obtain for the axial vector operator by simply replacing $\gamma_{\beta}$ by $\gamma_{\beta} \gamma_{5}$. 


\subsection{Antisymmetric tensor operators}

Now, together with its (partly) contracted operator $M_{\alpha}=x^{\beta} M_{\alpha \beta}$, we consider the following tensor operator 9

$$
\begin{aligned}
M_{\alpha \beta}(0, \kappa x) & =\bar{\psi}(0) \sigma_{\alpha \beta} U(0, \kappa x) \psi(\kappa x), \\
M_{\alpha}(0, \kappa x) & =\bar{\psi}(0) \sigma_{\alpha \beta} x^{\beta} U(0, \kappa x) \psi(\kappa x) .
\end{aligned}
$$

The corresponding local tensor operators are given by

$$
\begin{aligned}
M_{\alpha \beta \mu_{1} \ldots \mu_{n}} \equiv & \bar{\psi}(0) \sigma_{\alpha \beta} D_{\left\{\mu_{1}\right.} \ldots D_{\left.\mu_{n}\right\}} \psi(0) \\
= & \alpha_{n+1} \bar{\psi}(0) \sigma_{\alpha\{\beta} D_{\mu_{1}} \ldots D_{\left.\mu_{n}\right\}} \psi(0)+\beta_{n} \bar{\psi}(0) \sigma_{[\alpha \beta} D_{\left\{\mu_{1}\right]} \ldots D_{\left.\mu_{n}\right\}} \psi(0) \\
& +\ldots
\end{aligned}
$$

which also decompose into two parts being related to the Young patterns (ii) and (iii) and further contributions being partially antisymmetric with respect to the $\mu_{i}$ 's; $\alpha_{n+1}=2(n+1) /(n+2)$ is already known, and $\beta_{n}=3 n /(n+2)$ is the normalizing factor of $\mathcal{Y}_{[m]}$ with $[m]=(n, 1,1)$. These operators have mass dimension $n+3$; for $n=0$ the operator transforms as an antisymmetric tensor of rank 2 and therefore has $\operatorname{spin} j=1$, twist $\tau=2$. For $n>0$ these tensors are reducible. The corresponding Clebsch-Gordan series is given by

$$
\begin{aligned}
& ((1,0) \oplus(0,1)) \otimes\left(\left(\frac{n}{2}, \frac{n}{2}\right) \oplus\left(\frac{n-2}{2}, \frac{n-2}{2}\right) \oplus \ldots\right) \\
& \quad=\left(\left(\frac{n+2}{2}, \frac{n}{2}\right) \oplus\left(\frac{n}{2}, \frac{n+2}{2}\right)\right) \oplus 2\left(\frac{n}{2}, \frac{n}{2}\right) \oplus 2\left(\left(\frac{n-2}{2}, \frac{n}{2}\right) \oplus\left(\frac{n}{2}, \frac{n-2}{2}\right)\right) \oplus \ldots .
\end{aligned}
$$

Again, as in the case of the vector operators, eq. (3.18), this corresponds - for any fixed value of $n$ - to a finite twist decomposition $(\tau \geq 2)$. In the limit $x \rightarrow \tilde{x}$ only the first three (respectively two) terms survive to get

$$
\begin{aligned}
M_{\alpha \beta}(0, \kappa \tilde{x}) & =M_{\alpha \beta}^{\mathrm{tw} 2}(0, \kappa \tilde{x})+M_{\alpha \beta}^{\mathrm{tw} 3}(0, \kappa \tilde{x})+M_{\alpha \beta}^{\mathrm{tw} 4}(0, \kappa \tilde{x}) \\
M_{\alpha}(0, \kappa \tilde{x}) & =M_{\alpha}^{\mathrm{tw} 2}(0, \kappa \tilde{x})+M_{\alpha}^{\mathrm{tw} 3}(0, \kappa \tilde{x}) .
\end{aligned}
$$

The local tensors of symmetry class (ii) - the only ones being relevant for $M_{\alpha}(0, \kappa \tilde{x})$ - contribute to $\tau=2,3$, whereas those of symmetry class (iii) contribute to $\tau=3,4$. This may be seen as follows: In the case (ii) the trace terms being proportional to $\tilde{x}_{[\alpha} \sum_{i} g_{\beta] \mu_{i}}$ correspond to spin $j=n, n-1$ and, therefore, to twist $\tau=3,4$, and those being proportional to $x^{2} g_{\mu_{i} \mu_{j}}$ correspond to spin $j=n-1$ and twist $\tau=4$, but they disappear on the light-cone. In the case of symmetry class (iii) some of the latter contributions remain due to an additional derivation acting on $x^{2}$ (see eq. (3.80)). From this consideration it becomes obvious that

8 The contracted form of the antisymmetric tensor operator appears in applications, e.g., to the Drell-Yan process (see 20, 21]). Sometimes, especially in connection with expressions like $(\tilde{x} \gamma)(i \gamma D)$, also the (local) tensor operator with $\mathrm{i} \gamma_{\alpha} \gamma_{\beta}=\sigma_{\alpha \beta}+\mathrm{i} g_{\alpha \beta}$ has been used [23, 15] which, however, is not irreducible. We denoted these tensors by $M$ since they are related to the twist- 3 contribution of the quark mass in polarized deep inelastic scattering [31, 15]. 
the twist-2 and twist- 4 contributions of $M_{\alpha \beta}\left(0, \kappa_{1} \tilde{x}\right)$ are uniquely defined on the light-cone, whereas the twist- 3 contributions consist of two parts.

(A) Tensor operators of symmetry class (ii) and their contractions

Now we consider tensor operators (and their contractions with $x$ ) having symmetry class (ii) which is determined by the following standard tableaux:

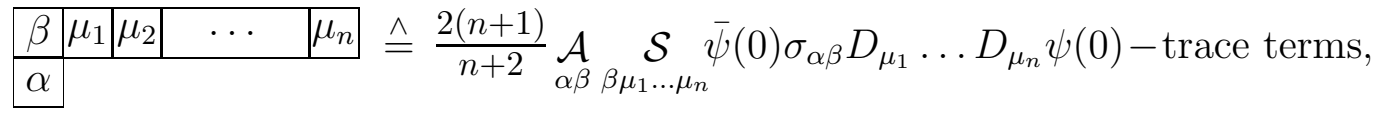

with normalizing factor $\alpha_{n+1}$. The corresponding twist-2 part transforms according to $\mathbf{T}\left(\frac{n+2}{2}, \frac{n}{2}\right) \oplus \mathbf{T}\left(\frac{n}{2}, \frac{n+2}{2}\right)$; it is given by:

$$
\begin{aligned}
M_{\alpha \beta \mu_{1} \ldots \mu_{n}}^{\mathrm{tw} 2}= & \frac{1}{n+2}\left(2 \bar{\psi}(0) \sigma_{\alpha \beta} D_{\left\{\mu_{1}\right.} \ldots D_{\left.\mu_{n}\right\}} \psi(0)\right. \\
& +\sum_{l=1}^{n} \bar{\psi}(0) \sigma_{\alpha\left\{\mu_{l}\right.} D_{\mu_{1}} \ldots D_{\mu_{l-1}} D_{|\beta|} D_{\mu_{l+1}} \ldots D_{\left.\mu_{n}\right\}} \psi(0) \\
& \left.-\sum_{l=1}^{n} \bar{\psi}(0) \sigma_{\beta\left\{\mu_{l}\right.} D_{\mu_{1}} \ldots D_{\mu_{l-1}} D_{|\alpha|} D_{\mu_{l+1}} \ldots D_{\left.\mu_{n}\right\}} \psi(0)\right)- \text { trace terms . }
\end{aligned}
$$

Of course, this representation is reducible with respect to $S O(1,3)$, because in that case it is possible to discriminate between the selfdual and antiselfdual part related to $\sigma_{\mu \nu}^{ \pm}$. However, in physical applications this is of no relevance, since only the orthochronous Lorentz group $O(1,3)$ is relevant and then parity connects them. After contraction with $x^{\mu_{1}} \ldots x^{\mu_{n}}$ we obtain:

$$
\begin{aligned}
M_{\alpha \beta n}^{\mathrm{tw} 2}(x) & =\frac{2}{n+2} \partial_{[\beta} \bar{\psi}(0) \sigma_{\alpha] \rho} x^{\rho}(x D)^{n} \psi(0)-\text { trace terms, } \\
M_{\alpha n+1}^{\mathrm{tw} 2}(x) & =\bar{\psi}(0) \sigma_{\alpha \rho} x^{\rho}(x D)^{n} \psi(0)-\text { trace terms }
\end{aligned}
$$

respectively.'T Proceeding in the same way as in the preceeding Subsections we sum up to obtain the nonlocal twist-2 operators:

$$
\begin{aligned}
& M_{\alpha \beta}^{\mathrm{tw} 2}(0, \kappa x)=2 \int_{0}^{1} \mathrm{~d} \lambda \lambda \partial_{[\beta}\left(M_{\alpha]}(0, \kappa \lambda x)-\text { trace terms }\right), \\
& M_{\alpha}^{\mathrm{tw} 2}(0, \kappa x)=\left(\delta_{\alpha}^{\mu}(x \partial)-x^{\mu} \partial_{\alpha}\right) \int_{0}^{1} \mathrm{~d} \lambda \lambda\left(M_{\mu}(0, \kappa \lambda x)-\text { trace terms }\right),
\end{aligned}
$$

respectively. Here, we remark that, due to the extra factor $x^{\beta}$ in eq. (3.49), also an extra factor $\lambda$ appears in the integrand of eqs. (3.58), (3.59) and, furthermore, that eq. (3.59) has exactly the same structure as in the case of vector operators.

Taking into account eq. (3.33) for the local traceless tensor $\stackrel{\circ}{M}_{\alpha n+1}$, the integral representations of $1 /(n+2)$ and of the beta function we arrive at the following

${ }^{9}$ Here, the same comments are in order as in footnote 7 . Let us remind that according to the definition of the antisymmetrization it holds $2 T_{[\mu \nu]}=T_{\mu \nu}-T_{\nu \mu}$. 
expression for the nonlocal traceless (vector) operator

$$
\begin{aligned}
\stackrel{\circ}{M}_{\alpha}(0, \kappa x)= & M_{\alpha}(0, \kappa x)+\sum_{k=1}^{\infty} \int_{0}^{1} \mathrm{~d} t\left(\frac{-x^{2}}{4}\right)^{k} \frac{\square^{k}}{k !(k-1) !}\left(\frac{1-t}{t}\right)^{k-1} M_{\alpha}(0, \kappa t x) \\
& -\left[x_{\alpha} \partial^{\mu}(x \partial)-\frac{1}{2} x^{2} \partial_{\alpha} \partial^{\mu}\right] \\
& \times \sum_{k=0}^{\infty} \int_{0}^{1} \mathrm{~d} \tau \tau \int_{0}^{1} \mathrm{~d} t t\left(\frac{-x^{2}}{4}\right)^{k} \frac{\square^{k}}{k ! k !}\left(\frac{1-t}{t}\right)^{k} M_{\mu}(0, \kappa \tau t x)
\end{aligned}
$$

From this the irreducible twist-2 operators are obtained as follows:

$$
\begin{aligned}
& M_{\alpha \beta}^{\mathrm{tw} 2}(0, \kappa x)=2 \int_{0}^{1} \mathrm{~d} \lambda \lambda \partial_{[\beta} \stackrel{\circ}{M}_{\alpha]}(0, \kappa \lambda x), \\
& M_{\alpha}^{\mathrm{tw} 2}(0, \kappa x)=\stackrel{\circ}{M}_{\alpha}(0, \kappa x) .
\end{aligned}
$$

To obtain the last equation use has been made of a partial integration; the result is in conformity with the twist-2 case of the vector operators (compare eq. (3.22) and the related remarks). Again, these operators are harmonic tensor functions:

$$
\begin{gathered}
\square M_{\alpha \beta}^{\mathrm{tw} 2}(0, \kappa x)=0, \quad \partial^{\alpha} M_{\alpha \beta}^{\mathrm{tw} 2}(0, \kappa x)=0, \quad \partial^{\beta} M_{\alpha \beta}^{\mathrm{tw} 2}(0, \kappa x)=0 \\
\square M_{\alpha}^{\mathrm{tw} 2}(0, \kappa x)=0, \quad \partial^{\alpha} M_{\alpha}^{\mathrm{tw} 2}(0, \kappa x)=0 ;
\end{gathered}
$$

the first set of equations, due to (3.61), is a consequence of the second ones.

For the projection onto the light-cone only the terms in eq. (3.60) with $k=1,2$ contribute. Now, observing the following equalities,

$$
\begin{aligned}
\int_{0}^{1} \mathrm{~d} \lambda \lambda \int_{0}^{1} \mathrm{~d} t f(\kappa \lambda t) & =\int_{0}^{1} \mathrm{~d} \lambda(1-\lambda) f(\kappa \lambda), \\
\int_{0}^{1} \mathrm{~d} \lambda \lambda \int_{0}^{1} \mathrm{~d} \tau \tau \int_{0}^{1} \mathrm{~d} t t f(\kappa \lambda \tau t) & =\frac{1}{2} \int_{0}^{1} \mathrm{~d} \lambda\left(\lambda \ln ^{2} \lambda\right) f(\kappa \lambda), \\
\int_{0}^{1} \mathrm{~d} \lambda \lambda \int_{0}^{1} \mathrm{~d} \tau \tau \int_{0}^{1} \mathrm{~d} t(1-t) f(\kappa \lambda \tau t) & =\int_{0}^{1} \mathrm{~d} \lambda\left(1-\lambda+\lambda \ln \lambda-\frac{\lambda}{2} \ln ^{2} \lambda\right) f(\kappa \lambda),
\end{aligned}
$$

we obtain for the nonlocal twist-2 light-cone tensor operator

$$
\begin{aligned}
M_{\alpha \beta}^{\mathrm{tw} 2}(0, \kappa \tilde{x})= & 2 \int_{0}^{1} \mathrm{~d} \lambda \lambda\left\{\delta_{[\alpha}^{\mu}\left(\partial_{\beta]}-\frac{1-\lambda}{2 \lambda} x_{\beta]} \square\right)-\frac{\ln ^{2} \lambda}{2} x_{[\alpha} \partial_{\beta]} \partial^{\mu}(1+x \partial)\right. \\
& \left.+\frac{1}{2}\left(\frac{1-\lambda}{\lambda}+\ln \lambda\right)\left(x_{[\alpha} \delta_{\beta]}^{\mu}+x^{\mu} x_{[\alpha} \partial_{\beta]}\right) \square\right\}\left.M_{\mu}(0, \kappa \lambda x)\right|_{x=\tilde{x}} .
\end{aligned}
$$

Performing the partial integrations which originate from $(x \partial) f(\lambda x)=\lambda \partial f / \partial \lambda$, and generalizing to arbitrary values of $\kappa_{i}$, we finally obtain

$$
\begin{aligned}
M_{\alpha \beta}^{\mathrm{tw} 2}\left(\kappa_{1} \tilde{x}, \kappa_{2} \tilde{x}\right) & =\left.2 \int_{0}^{1} \mathrm{~d} \lambda \lambda \partial_{[\beta} M_{\alpha]}\left(\kappa_{1} \lambda x, \kappa_{2} \lambda x\right)\right|_{x=\tilde{x}}-M_{\alpha \beta}^{\mathrm{higher}}\left(\kappa_{1} \tilde{x}, \kappa_{2} \tilde{x}\right),(3.6) \\
M_{\alpha \beta}^{\mathrm{higher}}\left(\kappa_{1} \tilde{x}, \kappa_{2} \tilde{x}\right) & =\left.\int_{0}^{1} \mathrm{~d} \lambda(1-\lambda)\left(2 x_{[\alpha} \partial_{\beta]} \partial^{\mu}-x_{[\alpha} \delta_{\beta]}^{\mu} \square\right) M_{\mu}\left(\kappa_{1} \lambda x, \kappa_{2} \lambda x\right)\right|_{x=\tilde{x}} .
\end{aligned}
$$


This last expression contains both twist- 3 and twist -4 contributions. After an explicit decomposition of $M_{\alpha \beta}^{\text {higher }}$ we get

$$
M_{\alpha \beta}^{\text {higher }}\left(\kappa_{1} \tilde{x}, \kappa_{2} \tilde{x}\right)=M_{\alpha \beta, a}^{\mathrm{tw} 3}\left(\kappa_{1} \tilde{x}, \kappa_{2} \tilde{x}\right)+M_{\alpha \beta, a}^{\mathrm{tw} 4}\left(\kappa_{1} \tilde{x}, \kappa_{2} \tilde{x}\right)
$$

with

$$
\begin{aligned}
M_{\alpha \beta, a}^{\mathrm{tw} 3}\left(\kappa_{1} \tilde{x}, \kappa_{2} \tilde{x}\right)= & -\left.\int_{0}^{1} \mathrm{~d} \lambda \frac{1-\lambda^{2}}{\lambda} x_{[\alpha} \partial_{\beta]} x^{[\mu} \partial^{\nu]} M_{\mu \nu}\left(\kappa_{1} \lambda x, \kappa_{2} \lambda x\right)\right|_{x=\tilde{x}} \\
M_{\alpha \beta, a}^{\mathrm{tw} 4}\left(\kappa_{1} \tilde{x}, \kappa_{2} \tilde{x}\right)= & -\int_{0}^{1} \mathrm{~d} \lambda\left\{\frac{(1-\lambda)^{2}}{\lambda} x_{[\alpha}\left(\delta_{\beta]}^{[\mu}(x \partial)-x^{[\mu} \partial_{\beta]}\right) \partial^{\nu]}\right. \\
& \left.+(1-\lambda) x_{[\alpha} \delta_{\beta]}^{[\mu} x^{\nu]} \square\right\}\left.M_{\mu \nu}\left(\kappa_{1} \lambda x, \kappa_{2} \lambda x\right)\right|_{x=\tilde{x}} .
\end{aligned}
$$

The twist-4 operator in eq. (3.72) consists of two several twist-4 operators with different prefactor.

Finally, performing the partial derivatives we obtain, especially for axial gauge,

$$
\begin{aligned}
& M_{\alpha \beta}^{\mathrm{tw} 2}\left(\kappa_{1} \tilde{x}, \kappa_{2} \tilde{x}\right)=2 \int_{0}^{1} \mathrm{~d} \lambda \lambda \bar{\psi}\left(\kappa_{1} y\right)\left\{\left[\sigma_{\alpha \beta}-y^{\rho} \sigma_{\rho[\alpha} \mathrm{D}_{\beta]}\right]\right. \\
& \left.\quad+\frac{1-\lambda}{\lambda}\left[2 y_{[\alpha} \sigma_{\beta] \mu} \mathrm{D}^{\mu}+y^{\rho} \sigma_{\rho \mu} y_{[\alpha} \mathrm{D}_{\beta]} \mathrm{D}^{\mu}+\frac{1}{2} y_{[\alpha} \sigma_{\beta] \rho} y^{\rho} \mathrm{D}^{2}\right]\right\}\left.\psi\left(\kappa_{2} y\right)\right|_{y=\lambda \tilde{x}}
\end{aligned}
$$

For the partially contracted nonlocal twist-2 light-cone operator we obtain

$$
M_{\alpha}^{\mathrm{tw} 2}(0, \kappa \tilde{x})=M_{\alpha}(0, \kappa x)+\left.\tilde{x}_{\alpha}\left(\partial_{\mu}(x \partial)-\frac{1}{2} x_{\mu} \square\right) \int_{0}^{1} \mathrm{~d} \lambda(\lambda \ln \lambda) M^{\mu}(0, \kappa \lambda x)\right|_{x=\tilde{x}},
$$

from which, after partial integration and generalization to arbitrary $\kappa_{i}$ we obtain

$$
\begin{aligned}
M_{\alpha}^{\mathrm{tw} 2}\left(\kappa_{1} \tilde{x}, \kappa_{2} \tilde{x}\right)= & \bar{\psi}\left(\kappa_{1} \tilde{x}\right) \sigma_{\alpha \rho} \tilde{x}^{\rho} U\left(\kappa_{1} \tilde{x}, \kappa_{2} \tilde{x}\right) \psi\left(\kappa_{2} \tilde{x}\right) \\
& +\left.\tilde{x}_{\alpha} \tilde{x}^{\rho} \partial^{\mu} \int_{0}^{1} \mathrm{~d} \lambda \lambda \bar{\psi}\left(\kappa_{1} \lambda x\right) \sigma_{\rho \mu} U\left(\kappa_{1} \lambda x, \kappa_{2} \lambda x\right) \psi\left(\kappa_{2} \lambda x\right)\right|_{x=\tilde{x}}
\end{aligned}
$$

and, correspondingly, in axial gauge

$$
M_{\alpha}^{\mathrm{tw} 2}\left(\kappa_{1} \tilde{x}, \kappa_{2} \tilde{x}\right)=\bar{\psi}\left(\kappa_{1} \tilde{x}\right) \sigma_{\alpha \rho} \tilde{x}^{\rho} \psi\left(\kappa_{2} \tilde{x}\right)+\left.\tilde{x}_{\alpha} \int_{0}^{1} \mathrm{~d} \lambda \bar{\psi}\left(\kappa_{1} y\right) y^{\rho} \sigma_{\rho \mu} \mathrm{D}^{\mu} \psi\left(\kappa_{2} y\right)\right|_{y=\lambda \tilde{x}} .
$$

Because the first term on the r.h. side equals $M_{\alpha}(0, \kappa \tilde{x})$ its twist- 3 part according to eq. (3.74) is given by the second term (up to a minus sign):

$$
M_{\alpha}^{\mathrm{tw} 3}\left(\kappa_{1} \tilde{x}, \kappa_{2} \tilde{x}\right)=\left.\tilde{x}_{\alpha} \tilde{x}^{\rho} \partial^{\mu} \int_{0}^{1} \mathrm{~d} \lambda \lambda \bar{\psi}\left(\kappa_{1} \lambda x\right) \sigma_{\mu \rho} U\left(\kappa_{1} \lambda x, \kappa_{2} \lambda x\right) \psi\left(\kappa_{2} \lambda x\right)\right|_{x=\tilde{x}} .
$$

\footnotetext{
${ }^{10}$ This result for $\kappa_{1}=1, \kappa_{2}=0$, and including $x^{2}$-terms, has been obtained earlier in Ref. [5]
} 
Its local components, which already have been used in [20, 25], are

$$
M_{\alpha n+1}^{\mathrm{tw} 3}=\frac{1}{n+2} \tilde{x}_{\alpha} \bar{\psi}(0) \sigma_{\mu \rho} \tilde{x}^{\rho}(\tilde{x} D)^{n} D^{\mu} \psi(0) .
$$

(B) Tensor operators of symmetry class (iii)

Now we consider the twist- 3 and twist -4 contributions originating from the (only possible) standard tableaux for the symmetry class (iii):

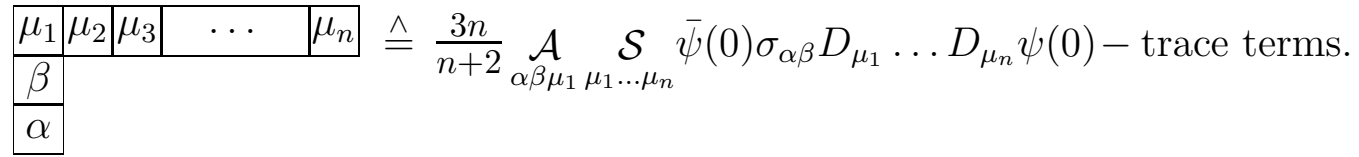

The corresponding traceless local tensor having twist $\tau=3$ and being contained in $\mathbf{T}\left(\frac{n}{2}, \frac{n}{2}\right)$ is given by (where $\beta_{n}=3 n /(n+2)$ has been choosen):

$$
\begin{gathered}
M_{\alpha \beta \mu_{1} \ldots \mu_{n}}^{\mathrm{tw} 3}=\frac{n}{n+2}\left(\bar{\psi}(0) \sigma_{\alpha \beta} D_{\left\{\mu_{1}\right.} \ldots D_{\left.\mu_{n}\right\}} \psi(0)-\bar{\psi}(0) \sigma_{\alpha \mu_{1}} D_{\{\beta} \ldots D_{\left.\mu_{n}\right\}} \psi(0)\right. \\
\left.+\bar{\psi}(0) \sigma_{\beta \mu_{1}} D_{\{\alpha} \ldots D_{\left.\mu_{n}\right\}} \psi(0)\right)- \text { trace terms. }
\end{gathered}
$$

Again, the prefactor $\beta_{n}$ could have been determined by the equality (compare eq. (3.30) )

$$
M_{\alpha \beta n}(x)=x^{\mu_{1}} \ldots x^{\mu_{n}}\left(\frac{2(n+1)}{n+2} \underset{\alpha \beta \beta \mu_{1} \ldots \mu_{n}}{\mathcal{S}} M_{\alpha \beta \mu_{1} \ldots \mu_{n}}+\frac{3 n}{n+2} \underset{\alpha \beta \mu_{1}}{\mathcal{A}} \underset{\mu_{1} \ldots \mu_{n}}{\mathcal{S}} M_{\alpha \beta \mu_{1} \ldots \mu_{n}}\right) .
$$

Now, contracting the expression (3.78) with $x^{\mu_{1}} \ldots x^{\mu_{n}}$ we obtain:

$$
\begin{aligned}
M_{\alpha \beta n}^{\mathrm{tw} 3, \mathrm{~b}}(x) & =x^{\mu_{1}} \ldots x^{\mu_{n}} M_{\alpha \beta \mu_{1} \ldots \mu_{n}}^{\mathrm{tw} 3 \mathrm{~b}} \\
& =\frac{1}{n+2}\left((x \partial) \delta_{[\beta}^{\rho}-2 x^{\rho} \partial_{[\beta}\right)\left(\bar{\psi}(0) \sigma_{\alpha] \rho}(x D)^{n} \psi(0)-\text { trace terms }\right) .
\end{aligned}
$$

Resumming these local terms gives the nonlocal twist-3 operator as follows

$$
M_{\alpha \beta, b}^{\mathrm{tw} 3}(0, \kappa x)=\int_{0}^{1} \mathrm{~d} \lambda \lambda\left((x \partial) \delta_{[\beta}^{\rho}-2 x^{\rho} \partial_{[\beta}\right) \stackrel{\circ}{M_{\alpha] \rho}}(0, \kappa \lambda x),
$$

where we introduced the traceless nonlocal operator $\stackrel{\circ}{M}_{\alpha \beta}(0, \kappa x)$ which results from $\bar{\psi}(0) \sigma_{\alpha \beta} U(0, \kappa x) \psi(\kappa x)$; it has to be determined by the conditions

$$
\square \stackrel{\circ}{M}_{\alpha \beta n}=0, \quad \stackrel{\circ}{\alpha}_{\alpha \beta n}=0, \quad \partial^{\beta} \stackrel{\circ}{M}_{\alpha \beta n}=0 .
$$

The solution of these equations reads (see Appendix B, eq. (B.11))

$$
\begin{aligned}
\stackrel{\circ}{M}_{\alpha \beta n}(x)= & \left\{\delta_{\alpha}^{\mu} \delta_{\beta}^{\nu}-\frac{2}{n(n+1)(n+2)}\left(x_{[\alpha} \partial_{\beta]} x^{[\mu} \partial^{\nu]}\right.\right. \\
& \left.\left.-\left[x_{[\alpha} \delta_{\beta]}^{[\mu} \partial^{\nu]}(x \partial)-\frac{1}{2} x^{2} \partial_{[\alpha} \delta_{\beta]}^{[\mu} \partial^{\nu]}\right](x \partial+2)\right)\right\} H_{n}^{(4)}\left(x^{2} \mid \square\right) M_{\mu \nu n}(x) .
\end{aligned}
$$


Using the integral representation of the additional factor $1 / n$ (the remaining factors of the denominator are taken together with $1 / n$ ! to get $1 /(n+2) !)$ and of the beta function we may sum up these local operators to the following expression:

$$
\begin{aligned}
\stackrel{\circ}{M}_{\alpha \beta}(0, \kappa x)= & M_{\alpha \beta}(0, \kappa x)+\sum_{k=1}^{\infty} \int_{0}^{1} \frac{\mathrm{d} t}{t}\left(\frac{-x^{2}}{4}\right)^{k} \frac{\square^{k}}{k !(k-1) !}\left(\frac{1-t}{t}\right)^{k-1} M_{\alpha \beta}(0, \kappa t x) \\
& -2\left\{x_{[\alpha} \partial_{\beta]} x^{[\mu} \partial^{\nu]}-\left[x_{[\alpha} \delta_{\beta]}^{[\mu} \partial^{\nu]}(x \partial)-\frac{1}{2} x^{2} \partial_{[\alpha} \delta_{\beta]}^{[\mu} \partial^{\nu]}\right](x \partial+2)\right\} \\
& \times \sum_{k=0}^{\infty} \int_{0}^{1} \frac{\mathrm{d} \tau}{\tau} \int_{0}^{1} \mathrm{~d} t t\left(\frac{-x^{2}}{4}\right)^{k} \frac{\square^{k}}{(k+1) ! k !}\left(\frac{1-t}{t}\right)^{k+1} M_{\mu \nu}(0, \kappa \tau t x) .
\end{aligned}
$$

Then, by construction, eq. (3.80) defines a nonlocal operator of twist-3. As is easily seen by partial integration it fulfils the following relation

$$
M_{\alpha \beta, b}^{\mathrm{tw} 3}(0, \kappa x)=\stackrel{\circ}{M}_{\alpha \beta}(0, \kappa x)-M_{\alpha \beta}^{\mathrm{tw} 2}(0, \kappa x)
$$

and, because of eqs. (3.63) and (3.81) it is a harmonic tensor operator.

Now, to determine the corresponding LC-operator we use eq. (3.84). Because $M_{\alpha \beta}^{\mathrm{tw} 2}(0, \kappa \tilde{x})$ is already known we only have to determine $\stackrel{\circ}{M}_{\alpha \beta}(0, \kappa \tilde{x})$ for which only the terms with $k=0,1$ are relevant. Using the equalities

$$
\begin{aligned}
\int_{0}^{1} \frac{\mathrm{d} \tau}{\tau} \int_{0}^{1} \mathrm{~d} t(1-t) f(\kappa \tau t) & =\int_{0}^{1} \frac{\mathrm{d} t}{t} \frac{(1-t)^{2}}{2} f(\kappa t), \\
\int_{0}^{1} \frac{\mathrm{d} \tau}{\tau} \int_{0}^{1} \mathrm{~d} t \frac{(1-t)^{2}}{t} f(\kappa \tau t) & =-\int_{0}^{1} \frac{\mathrm{d} t}{t}\left(\frac{(1-t)^{2}}{2}+(1-t)+\ln t\right) f(\kappa t),
\end{aligned}
$$

we finally obtain:

$$
\begin{aligned}
& \stackrel{\circ}{M}_{\alpha \beta}(0, \kappa \tilde{x})=M_{\alpha \beta}(0, \kappa \tilde{x})-\left[x_{[\alpha} \partial_{\beta]} x^{[\mu} \partial^{\nu]}-x_{[\alpha} \delta_{\beta]}^{[\mu} \partial^{\nu]}(x \partial)(x \partial+2)\right] \\
& \quad \times\left.\int_{0}^{1} \frac{\mathrm{d} \lambda}{\lambda}\left((1-\lambda)^{2}+\frac{1}{8}\left\{(1-\lambda)^{2}+2(1-\lambda)+2 \ln \lambda\right\} x^{2} \square\right) M_{\mu \nu}(0, \kappa \lambda x)\right|_{x=\tilde{x}},
\end{aligned}
$$

which, after performing the partial integrations due to $(x \partial) f(\lambda x)=\lambda \partial f(\lambda x) / \partial \lambda$, leads to the expression:

$$
\begin{aligned}
\stackrel{\circ}{M}_{\alpha \beta}(0, \kappa \tilde{x})=M_{\alpha \beta}(0, \kappa \tilde{x})- & \int_{0}^{1} \frac{\mathrm{d} \lambda}{\lambda}\left\{(1-\lambda)^{2} x_{[\alpha} \partial_{\beta]} x^{[\mu} \partial^{\nu]}\right. \\
& \left.-2 \lambda x_{[\alpha} \delta_{\beta]}^{[\mu} \partial^{\nu]}+(1-\lambda) x_{[\alpha} \delta_{\beta]}^{[\mu} x^{\nu]} \square\right\} M_{\mu \nu}(0, \kappa \lambda x)_{\mid x=\tilde{x}} .
\end{aligned}
$$

Now, inserting eqs. (3.88) and (3.70) into eq. (3.84) we obtain:

$$
\begin{aligned}
& M_{\alpha \beta, b}^{\mathrm{tw} 3}(0, \kappa \tilde{x})=M_{\alpha \beta}(0, \kappa \tilde{x})-\left.2 \int_{0}^{1} \mathrm{~d} \lambda \lambda \partial_{[\beta} M_{\alpha]}(0, \kappa \lambda x)\right|_{x=\tilde{x}} \\
& \quad-\left.\int_{0}^{1} \frac{\mathrm{d} \lambda}{\lambda}\left\{\left(1-\lambda^{2}\right)\left(x_{[\alpha} \delta_{\beta]}^{[\mu} x^{\nu]} \square+x_{[\alpha} \partial_{\beta]} x^{[\mu} \partial^{\nu]}\right)-2 \lambda^{2} x_{[\alpha} \delta_{\beta]}^{[\mu} \partial^{\nu]}\right\} M_{\mu \nu}(0, \kappa \lambda x)\right|_{x=\tilde{x}} .
\end{aligned}
$$


Let us write this for arbitrary $\kappa_{i}$ in the form

$$
\begin{aligned}
M_{\alpha \beta, b}^{\mathrm{tw} 3}\left(\kappa_{1} \tilde{x}, \kappa_{2} \tilde{x}\right)= & \left.\int_{0}^{1} \mathrm{~d} \lambda \lambda\left((x \partial) \delta_{[\beta}^{\nu}-2 x^{\nu} \partial_{[\beta}\right) M_{\alpha] \nu}\left(\kappa_{1} \lambda x, \kappa_{2} \lambda x\right)\right|_{x=\tilde{x}} \\
& -M_{\alpha \beta, b}^{\mathrm{tw} 4}\left(\kappa_{1} \tilde{x}, \kappa_{2} \tilde{x}\right),
\end{aligned}
$$

where the twist -4 part is determined by the trace terms, namely

$$
\begin{aligned}
M_{\alpha \beta, b}^{\mathrm{tw} 4}\left(\kappa_{1} \tilde{x}, \kappa_{2} \tilde{x}\right)= & -\int_{0}^{1} \mathrm{~d} \lambda \frac{1-\lambda^{2}}{\lambda}\left\{x_{[\alpha}\left(\delta_{\beta]}^{[\mu}(x \partial)-x^{[\mu} \partial_{\beta]}\right) \partial^{\nu]}\right. \\
& \left.-x_{[\alpha} \delta_{\beta]}^{[\mu} x^{\nu]} \square\right\}\left.M_{\mu \nu}\left(\kappa_{1} \lambda x, \kappa_{2} \lambda x\right)\right|_{x=\tilde{x}} .
\end{aligned}
$$

The full twist-4 operator gets from eqs. (3.72) and (3.91)

$$
\begin{aligned}
& M_{\alpha \beta}^{\mathrm{tw} 4}\left(\kappa_{1} \tilde{x}, \kappa_{2} \tilde{x}\right)=M_{\alpha \beta, a}^{\mathrm{tw} 4}\left(\kappa_{1} \tilde{x}, \kappa_{2} \tilde{x}\right)+M_{\alpha \beta, b}^{\mathrm{tw} 4}\left(\kappa_{1} \tilde{x}, \kappa_{2} \tilde{x}\right) \\
& \quad=\left.\int_{0}^{1} \mathrm{~d} \lambda \frac{1-\lambda}{\lambda}\left\{x_{[\alpha} \delta_{\beta]}^{[\mu} x^{\nu]} \square-2 x_{[\alpha}\left(\delta_{\beta]}^{[\mu}(x \partial)-x^{[\mu} \partial_{\beta]}\right) \partial^{\nu]}\right\} M_{\mu \nu}\left(\kappa_{1} \lambda x, \kappa_{2} \lambda x\right)\right|_{x=\tilde{x}} .
\end{aligned}
$$

The complete twist-3 operator obtains from eq. (3.90) and the trace terms with twist -3 of the twist -2 operator eq. (3.71):

$$
\begin{aligned}
& M_{\alpha \beta}^{\mathrm{tw} 3}\left(\kappa_{1} \tilde{x}, \kappa_{2} \tilde{x}\right)=M_{\alpha \beta, a}^{\mathrm{tw} 3}\left(\kappa_{1} \tilde{x}, \kappa_{2} \tilde{x}\right)+M_{\alpha \beta, b}^{\mathrm{tw} 3}\left(\kappa_{1} \tilde{x}, \kappa_{2} \tilde{x}\right) \\
& \quad=\int_{0}^{1} \mathrm{~d} \lambda\left\{\lambda\left((x \partial) \delta_{[\beta}^{\nu}-2 x^{\nu} \partial_{[\beta}\right) \delta_{\alpha]}^{\mu}-\frac{1-\lambda^{2}}{\lambda}\left(x_{[\alpha} \partial_{\beta]} x^{[\mu} \partial^{\nu]}\right.\right. \\
& \left.\left.\quad+x_{[\alpha}\left(\delta_{\beta]}^{[\mu}(x \partial)-x^{[\mu} \partial_{\beta]}\right) \partial^{\nu]}-x_{[\alpha} \delta_{\beta]}^{[\mu} x^{\nu]} \square\right)\right\}\left.M_{\mu \nu}\left(\kappa_{1} \lambda x, \kappa_{2} \lambda x\right)\right|_{x=\tilde{x}} .
\end{aligned}
$$

This completes the twist decomposition of the antisymmetric tensor operators.

\section{Applications to distribution amplitudes and generalization to conformal LC operators}

In this Section we like to point out one of the virtues of the twist decomposition which have been given in Section 3 by considering nonforward matrix elements of the nonlocal LC operators with definite twist. Furthermore, we introduce the generalization of the twist decomposition to conformal light-cone operators.

\subsection{Matrix elements of LC operators with definite twist}

As it became obvious there exist relations between nonlocal operators of the same twist but different tensor structure, e.g., between the vector and scalar operators, cf. Eqs. (3.23) and (3.13) as well as Eqs. (3.61) and (3.62), and also of different twist and same tensor structure, cf. Eqs. (3.36) and (3.23). This, of course, leads 
to relations between the related distribution amplitudes obtained from the (nonforward) matrix elements.

The nonforward matrix elements of light-ray operators with definite twist between the hadron states $\left|p_{i}, S_{i}\right\rangle$ with momenta $p_{i}$ and spin $S_{i}, i=1,2$, appear as nonperturbative inputs of various hard scattering processes. Phenomenologically these quantities will be represented by partition functions (in the forward case) resp. distribution amplitudes. Because of translation invariance,

$$
\left\langle p_{2}\left|O^{\Gamma}\left(\kappa_{1} x, \kappa_{2} x\right)\right| p_{1}\right\rangle=e^{i \kappa_{+} x p_{-}}\left\langle p_{2}\left|O^{\Gamma}\left(-\kappa_{-} x, \kappa_{-} x\right)\right| p_{1}\right\rangle,
$$

it is completely sufficient to consider matrix elements of the 'centered' operators $O^{\Gamma}(-\kappa \tilde{x}, \kappa \tilde{x})$. Their Fourier transforms with respect to $\kappa \tilde{x} p_{+}$and $\kappa \tilde{x} p_{-}$define the above mentioned distribution amplitudes. In the following it is useful - as it was the case for the twist decomposition - to study the nonlocal operators temporarily for arbitrary values of $x$. Then, the distribution amplitudes on the light-cone are obtained as limiting case $x \rightarrow \tilde{x}$.

First, we consider the scalar twist-2 quark operators (3.11) between hadron states $\left|p_{i}, S_{i}\right\rangle$ and, in addition, we perform a kinematical decomposition of these matrix elements. If the equation of motion for the external hadron states are taken into account, then there remain only two independent distribution amplitudes which may be choosen in the following way $(u(p) \equiv u(p, s)$ is the free hadronic Dirac spinor and, to simplify notations, $\sigma_{\mu \nu} x^{\mu} p_{-}^{\nu}$ is denoted by $\left.\left(x \sigma p_{-}\right)\right)$:

$$
\begin{aligned}
\left\langle p_{2}\left|O^{\mathrm{tw} 2}(-\kappa x, \kappa x)\right| p_{1}\right\rangle & =\tilde{g}^{(2)}\left(\kappa x p_{+}, \kappa x p_{-}, \kappa^{2} x^{2}, p_{1} p_{2}, \mu^{2}\right) \bar{u}\left(p_{2}\right)(x \gamma) u\left(p_{1}\right) \\
& +\tilde{h}^{(2)}\left(\kappa x p_{+}, \kappa x p_{-}, \kappa^{2} x^{2}, p_{1} p_{2}, \mu^{2}\right) M^{-1} \bar{u}\left(p_{2}\right)\left(x \sigma p_{-}\right) u\left(p_{1}\right) .
\end{aligned}
$$

Because of eq. (3.23) the twist-2 vector operator is related to this kinematic decomposition:田

$$
\begin{aligned}
& \left\langle p_{2}\left|O_{\mu}^{\mathrm{tw} 2}(-\kappa x, \kappa x)\right| p_{1}\right\rangle=\int_{0}^{1} d \lambda \partial_{\mu}^{x}\left\langle p_{2}\left|O^{\mathrm{tw} 2}(-\kappa \lambda x, \kappa \lambda x)\right| p_{1}\right\rangle \\
& =\int_{0}^{1} d \lambda \partial_{\mu}^{x}\left[\tilde{g}^{(2)}\left(\kappa \lambda x p_{+}, \kappa \lambda x p_{-}, \kappa^{2} \lambda^{2} x^{2}, p_{1} p_{2}, \mu^{2}\right) \bar{u}\left(p_{2}\right)(x \gamma) u\left(p_{1}\right)\right. \\
& \left.\quad+\tilde{h}^{(2)}\left(\kappa \lambda x p_{+}, \kappa \lambda x p_{-}, \kappa^{2} \lambda^{2} x^{2}, p_{1} p_{2}, \mu^{2}\right) M^{-1} \bar{u}\left(p_{2}\right)\left(x \sigma p_{-}\right) u\left(p_{1}\right)\right] .
\end{aligned}
$$

The application of this relationship between scalar and vector operators to the virtual Compton scattering has been considered in detail in [8]. Especially, it has been shown that any of the physical results about the twist-2 vector operator is already contained in the twist-2 scalar operator and its distribution amplitudes. In order to indicate this roughly the Fourier transformations of $g$ and $h$ - jointly being denoted by $f$ - with respect to $\kappa x p_{ \pm}$as well as $\kappa^{2} x^{2}$ are introduced,

$\tilde{f}\left(\kappa x p_{+}, \kappa x p_{-}, \kappa^{2} x^{2}\right)=\int_{-1}^{+1} d z_{+} d z_{-} e^{-i \kappa\left(\left(x p_{+}\right) z_{+}+\left(x p_{-}\right) z_{-}\right)} \int d q e^{-i \kappa x q} f\left(z_{+}, z_{-}, Q^{2}\right)$,

${ }^{11}$ Here, it is necessary to remark that for the consideration of anomalous dimensions and evolution equations of amplitudes it is mandatory to use the operators with general $\kappa$-values. 
where unaffected arguments have been omitted. Then the $\lambda$-integration will be performed leading to

$$
\begin{aligned}
& \left\langle p_{2}\left|O_{\mu}^{\mathrm{tw} 2}(-\kappa x, \kappa x)\right| p_{1}\right\rangle=\int_{-1}^{+1} d z_{+} d z_{-} e^{-i \kappa\left(\left(x p_{+}\right) z_{+}+\left(x p_{-}\right) z_{-}\right)} \int d q e^{-i \kappa x q} \\
& \left\{G\left(z_{+}, z_{-}, Q^{2}\right)\left[\delta_{\mu}^{\lambda}-i \kappa g_{\mu \nu}\left(p_{+}^{\nu} z_{+}+p_{-}^{\nu} z_{+}+q^{\nu}\right) x^{\lambda}\right] \bar{u}\left(p_{2}\right) \gamma_{\lambda} u\left(p_{1}\right)\right. \\
& \quad+H\left(z_{+}, z_{-}, Q^{2}\right)\left[\delta_{\mu}^{\lambda}-i \kappa g_{\mu \nu}\left(p_{+}^{\nu} z_{+}+p_{-}^{\nu} z_{+}+q^{\nu}\right) x^{\lambda}\right] M^{-1} \bar{u}\left(p_{2}\right)\left(\sigma_{\lambda \kappa} p_{-}^{\kappa}\right) u\left(p_{1}\right)
\end{aligned}
$$

where, again $F$ being either $G$ or $H$,

$$
F\left(z_{+}, z_{-}, Q^{2}\right)=\int_{0}^{1} \frac{d \lambda}{\lambda^{6}} f\left(\frac{z_{+}}{\lambda}, \frac{z_{-}}{\lambda}, \frac{Q^{2}}{\lambda^{2}}\right) \Theta\left(\lambda-\left|z_{+}\right|\right) \Theta\left(\lambda-\left|z_{-}\right|\right) .
$$

As has been proven in [8] if eq. (4.4) is used in the nonlocal light-cone expansion of the product of the electromagnetic currents appearing in the scattering amplitude of the virtual Compton scattering the latter will be expressed by the double variable distributions $G, H$, and the evolution of these amplitudes is governed by the anomalous dimensions of the twist-2 nonlocal scalar operators.

In the case of twist- 3 vector operators the situation is more complicated. However, the defining equation (3.36) may be rewritten somewhat and after partial integration we obtain a relation which is similar to eq. (3.84):

$$
\begin{aligned}
O_{\beta}^{\mathrm{tw}}(-\kappa x, \kappa x) & =\left(\delta_{\beta}^{\alpha}(x \partial+1)-\partial_{\beta} x^{\alpha}\right) \int_{0}^{1} \mathrm{~d} \lambda \stackrel{\circ}{O}_{\alpha}(-\kappa \lambda x, \kappa \lambda x) \\
& =\stackrel{\circ}{O}_{\beta}(-\kappa x, \kappa x)-O_{\beta}^{\mathrm{tw} 2}(-\kappa x, \kappa x) .
\end{aligned}
$$

Furthermore, from eq. (3.84) we obtain the relation

$$
x^{\beta} O_{\beta}^{\mathrm{tw} 3}(-\kappa x, \kappa x)=0 .
$$

Both equations restrict the nonforward matrix elements of the twist- 3 vector operators. From the last equation we get

$$
\begin{aligned}
\left\langle p_{2}\left|O_{\mu}^{\mathrm{tw} 3}(-\kappa x, \kappa x)\right| p_{1}\right\rangle= & \left(\delta_{\mu}^{\alpha} x^{\beta}-\delta_{\mu}^{\beta} x^{\alpha}\right)\left[\tilde{g}_{\alpha}^{(3)}\left(\kappa x p_{+}, \kappa x p_{-}, \kappa^{2} x^{2}\right) \bar{u}\left(p_{2}\right) \gamma_{\beta} u\left(p_{1}\right)\right. \\
& \left.\left.+\tilde{h}_{\alpha}^{(3)}\left(\kappa x p_{+}, \kappa x p_{-}, \kappa^{2} x^{2}\right) M^{-1} \bar{u}\left(p_{2}\right) \sigma_{\beta \nu} p_{-}^{\nu}\right) u\left(p_{1}\right)\right] \\
& +{\tilde{h^{\prime}}}_{\alpha}^{(3)}\left(\kappa x p_{+}, \kappa x p_{-}, \kappa^{2} x^{2}\right) \bar{u}\left(p_{2}\right) \sigma_{\mu \nu} x^{\nu} u\left(p_{1}\right)
\end{aligned}
$$

where the functions $\tilde{g}_{\mu}^{(3)}, \tilde{h}_{\mu}^{(3)}$ and ${\tilde{h^{\prime}}}_{\mu}^{(3)}$, because of eq. (4.5), are related to $\tilde{g}^{(2)}$ and $\tilde{h}^{(2)}$ and the nonforward matrix elements of $\stackrel{\circ}{O}_{\alpha}(-\kappa x, \kappa x)$; however, the latter can not be determined so easily.

In the case of twist- 2 tensor operators $M_{\alpha \beta}^{\mathrm{tw} 2}(0, \kappa x)$ the situation is more simple since, because of eqs. (3.61) and (3.62), we have the relation

$$
M_{\alpha \beta}^{\mathrm{tw} 2}(-\kappa x, \kappa x)=2 \int_{0}^{1} \mathrm{~d} \lambda \lambda \partial_{[\beta} M_{\alpha]}^{\mathrm{tw} 2}(-\kappa \lambda x, \kappa \lambda x) .
$$


From this equation, being similar to (3.23), relations may be derived which are analogous to eqs. (4.2) and (4.3). Physical consequences of this circumstance have not been drawn up to now. The situation in the case of twist-3 tensor operators is similar to the twist- 3 vector operators above - at least concerning that part which obtains from symmetry type (ii).

\subsection{Conformal nonlocal LC operators with definite twist}

As is well known the diagonalization of the anomalous dimensions which is essential for the solution of the evolution equations of the distribution amplitudes at least in one-loop order - is obtained by using conformal light-cone operators. Therefore, the results of this paper have to be generalized to conformal operators. This, however, is easily achieved since the light-cone operators $O_{\Gamma}\left(\kappa_{1} \tilde{x}, \kappa_{2} \tilde{x}\right)$ considered in Section 3 are related to the conformal ones by the following integral representation [10, 11]:12]

$$
\begin{aligned}
O_{\Gamma}^{\operatorname{conf}}(\kappa, t ; \tilde{x}) & =\partial_{(i \kappa)} \int \frac{d \rho}{2 \pi} O_{\Gamma}((\kappa-(1-t) \rho) \tilde{x},(\kappa+(1+t) \rho) \tilde{x}) \\
O_{\Gamma}\left(\kappa_{1} \tilde{x}, \kappa_{2} \tilde{x}\right) & =\int d t O_{\Gamma}^{\operatorname{conf}}\left(\kappa=\kappa_{+}+\kappa_{-} t, t ; \tilde{x}\right) .
\end{aligned}
$$

The coefficient functions $F_{\Gamma}^{\text {conf }}\left(x^{2}, \kappa, t\right)$ in the conformal operator product expansion, which is analogous to equ. (1.3), are given by

$$
F_{\Gamma}\left(x^{2}, \tilde{x} p_{1}, \tilde{x} p_{2}\right)=\int d \rho \int d t e^{i \kappa\left(\tilde{x} p_{+}\right)} \delta\left(t-\frac{\tilde{x} p_{-}}{\tilde{x} p_{+}}\right) F_{\Gamma}^{\mathrm{conf}}\left(x^{2}, \kappa, t\right)
$$

Let us remark that

$$
e^{i \kappa\left(\tilde{x} p_{+}\right)}\left|\tilde{x} p_{-}\right| \delta\left(\tilde{x} p_{-} t-\tilde{x} p_{+}\right)=: \partial_{(i \kappa)} \int \frac{d \rho}{2 \pi} e^{i \kappa\left(\tilde{x} p_{+}\right)+i \rho\left[\left(\tilde{x} p_{+}\right) t-\left(\tilde{x} p_{-}\right)\right]}
$$

defines $\partial_{(i \kappa)}$ (for a precise mathematical definition see [11]).

The corresponding local operators are determined according to

$$
\begin{aligned}
O_{\Gamma, k \ell}^{\text {conf }} & =i^{\ell} \partial^{\ell} /\left.\partial \kappa^{\ell} \int d t C_{k}^{\nu}(t) O_{\Gamma}^{\text {conf }}(\kappa, t ; \tilde{x})\right|_{\kappa=0} \\
& =\left.\bar{\psi}\left(x_{1}\right) \Gamma\left(i \tilde{x} \partial_{+}\right)^{\ell} C_{k}^{\nu}\left(\frac{\tilde{x} D_{-}}{\tilde{x} \partial_{+}}\right) \psi\left(x_{2}\right)\right|_{x_{1}=x_{2}=0} \quad \text { with } \quad \ell \leq k, \\
O_{\Gamma}^{\operatorname{conf}}(\kappa, t ; \tilde{x}) & =\sum_{k, \ell=0}^{\infty}(-1)^{\ell} \frac{\kappa^{\ell}}{\ell !} N_{k}^{\nu}\left(1-t^{2}\right)^{\nu-\frac{1}{2}} C_{k}^{\nu}(t) O_{\Gamma, k \ell}^{\operatorname{conf}}\left(\kappa_{1} \tilde{x}, \kappa_{2} \tilde{x}\right),
\end{aligned}
$$

${ }^{12}$ To be more precise, the operators $O_{\Gamma}^{\text {conf }}(\kappa, t ; \tilde{x})$ obviously are scale invariant, and the local conformal operators according to eqs. 4.12$)-(4.14)$ are directly related to them; see also the discussion of eq. (4.15) below. Especially, they do not have definite conformal spin. However, the latter may be obtained by a more involved resummation of the local conformal operators which does not affect their twist decomposition. For a more detailed discussion of nonlocal conformal operators see Chapter 3 of Ref. [4]. 
where

$$
N_{k}^{\nu}=2^{1-2 \nu} \frac{\Gamma^{2}\left(\frac{1}{2}\right) \Gamma(2 \nu+k)}{\Gamma^{2}(\nu)(\nu+k) k !}
$$

is the normalization factor which appears in the completeness and orthogonality relation of the Gegenbauer polynomials $C_{k}^{\nu}(t)$ of order $k$, index $\nu=d+s-\frac{1}{2}$ with $d$ and $s$ being the (canonical) dimension and the spin of the fields $\psi$, respectively:

$$
\sum_{k=0}^{\infty} \frac{\left(1-t^{2}\right)^{\nu-\frac{1}{2}}}{N_{k}^{\nu}} C_{k}^{\nu}(t) C_{k}^{\nu}\left(t^{\prime}\right)=\delta\left(t-t^{\prime}\right), \quad \int_{-1}^{+1} d t\left(1-t^{2}\right)^{\nu-\frac{1}{2}} C_{k}^{\nu}(t) C_{k^{\prime}}^{\nu}(t)=N_{k}^{\nu} \delta_{k k^{\prime}}
$$

Let us remark that in order to verify the relation (4.14) between the local and the nonlocal conformal operators the restriction $\ell \geq k$ must be annuled and the resulting function has to be analytically continued with respect to the argument $t$ which, from the orthogonality relation, is restricted to the intervall $|t| \leq 1$.

The relations (4.9) and (4.10) between the (usual) nonlocal operators and the conformal ones do not suffer from this drewback. Therefore, it is obvious that the nonlocal conformal light-cone operators of definite twist are obtained from the original scalar, vector and tensor operators of definite twist, which have been determined in Chapter 3, by simply inserting them into eq. (4.9). This may be read off also from eq. (4.13) if one observes that

$$
\left(i \tilde{x} \partial_{+}\right)^{\ell} C_{k}^{\nu}\left(\frac{\tilde{x} D_{-}}{\tilde{x} \partial_{+}}\right)=\left(i \tilde{x} \partial_{+}\right)^{\ell-k} \sum_{r=0}^{k} e_{r}^{k}\left(i \tilde{x} \partial_{+}\right)^{r}\left(\tilde{x} D_{-}\right)^{k-r}
$$

where $e_{r}^{k}$ are the expansion coefficients of the Gegenbauer polynomials, is a sum of terms containing the same number of differential operators which are multiplied by $\tilde{x}^{\mu_{1}} \ldots \tilde{x}^{\mu_{\ell}}$. Again, the fact will be used that the decomposition of a Lorentz tensor into irreducible ones does not depend on how the "internal" symmetry of that tensor is build up. The twist decompositions of each individual term in the above expansion (4.15) will be resummed to get the twist decomposition of the local conformal operators. From this the twist decomposition of the nonlocal conformal operators obtains.

The general result will be

$$
O_{\Gamma}^{\mathrm{conf}}(\kappa, t ; \tilde{x})=O_{\Gamma}^{\mathrm{conf}, \mathrm{tw}^{2}}(\kappa, t ; \tilde{x})+O_{\Gamma}^{\mathrm{conf}, \mathrm{tw} 3}(\kappa, t ; \tilde{x})+O_{\Gamma}^{\mathrm{conf}, \mathrm{tw} 4}(\kappa, t ; \tilde{x})
$$

with

$$
O_{\Gamma}^{\text {conf, } \tau}(\kappa, t ; \tilde{x})=\partial_{(i \kappa)} \int \frac{d \rho}{2 \pi} O_{\Gamma}^{\tau}(\kappa-(1-t) \rho, \kappa+(1+t) \rho)
$$

where $O_{\Gamma}^{\tau}\left(\kappa_{1}, \kappa_{2}\right)$ has to be taken from the expressions determined in Subsections 3.1 - 3.3, namely Eqs. (3.12) in the scalar case, (3.25), (3.26), (3.41) and (3.46) in the vector case, as well as (3.68), (3.69), (3.88) and (3.89) in the tensor case. Here, it should be remarked that for the generalization to the conformal operators it was essential that the twist decomposition holds for arbitrary values of $\kappa_{i}$ and not only for special ones like $\left(\kappa_{1}, \kappa_{2}\right)=(-\kappa,+\kappa)$. 


\section{Conclusions and Outlook}

In this paper we introduced an algorithm to decompose bilocal light-ray operators into operators of well-defined geometric twist and applied it to the bilocal quark operators which appear, e.g., in virtual Compton scattering in the generalized Bjorken region. To achieve this we made a Taylor expansion with respect to both arbitrary space-time arguments of the nonlocal operators to get the related local tensor operators. Group theoretically the method is based on the (complete) reduction of the corresponding tensor representations of the orthrochronous Lorentz group into irreducible representations. Then we resummed the local operators of definite twist to nonlocal ones. After projection onto the light-cone the (finite) twist decomposition of scalar, vector and antisymmetric tensor operators results. Thereby we were able to determine the trace terms of the nonlocal operators explicitly. Because of the fact that the "internal" Lorentz indices of the local operators have to be contracted by the (symmetric) product of the coordinates $x$ the nonlocal operators with definit twist are harmonic tensor functions. The polynomial basis of these tensor functions has been determined in Appendix B for the scalar, vector and (second rank) antisymmetric tensor case. If necessary, this could be extended also to symmetric tensors - which are of interest if gluon operators have to be considered - and to tensors of higher rank.

There are many reasons why these operators had to be determined. First of all, the more principal one, to make the twist decomposition rigorous and explicit. This is required if the renormalization of the light-ray operators should be considered on a safe basis. Up to now the anomalous dimensions of the light-cone operators have been determined without checking it explicitly also for the trace terms. As long as only leading orders are of interest this may be acceptable. However, if higher perturbational orders come into the play one gets serious troubles in determining the anomalous dimensions of, say, twist-4 operators because trace terms contribute essentially.

Another point of principal interest, but also with practical consequences, obtains if, as partly has been done in Subsection 4.1, matrix elements of light-cone operators with definite twist are considered. Whereas up to now the amplitudes related to the twist-2 vector operator had to be projected onto the scalar case by an additional contraction with $x$ it is now possible to extract more detailed information (see [8]). Namely, having determined the explicit twist decomposition it is possible, at least in principle, to relate the distribution amplitudes for vector and tensor operators. Furthermore, additional informations may be obtained also for operators of higher twist and different relationships between them might be derived. This, of course, has to be considered in more detail.

A further problem, which however appears to be much easier to solve, is to show how the geometrical twist decomposition which has been given in this paper relates to the dynamical twist decomposition of parton distributions given, e.g., by Jaffe and Ji [20]; see also [21]. Of course, it is to be expected that both decompositions coincide in the leading terms, but differ at higher orders. This difference between 
'geometric' and 'dynamic' twist decomposition has been discussed in [6] using the framework of Ref. [1; in fact, the consequences of this difference for twist-3 (in the dynamical sense) distribution amplitudes of vector mesons are demonstrated in full detail.

As we have seen, a simple reduction to the scalar case is impossible for the twist -3 operators. This will be of interest for the structure function $g_{2}$ in polarized deep inelastic scattering. The corresponding twist- 3 tensor operators transforming according the the same symmetry class (ii) are given by 15 [3:

$$
\begin{aligned}
O_{\mu}^{\mathrm{tw} 3}\left(\kappa_{1} x, \kappa_{2} x\right) & =i\left(\delta_{\mu}^{\nu}(x \partial)-x^{\nu} \partial_{\mu}\right) \int_{0}^{1} d u \bar{\psi}\left(\kappa_{1} u x\right) \gamma_{\nu} \gamma_{5} \psi\left(\kappa_{2} u x\right), \\
M_{\mu}^{\mathrm{tw} 3}\left(\kappa_{1} x, \kappa_{2} x\right) & =m\left(\delta_{\mu}^{\nu}(x \partial)-x^{\nu} \partial_{\mu}\right) \int_{0}^{1} d u u \bar{\psi}\left(\kappa_{1} u x\right) x^{\rho} \sigma_{\rho \nu} \gamma_{5} \psi\left(\kappa_{2} u x\right), \\
S_{\mu}^{+}\left(\kappa_{1} x, \kappa_{2} x\right) & =\int_{0}^{1} d u u \bar{\psi}\left(\kappa_{1} u x\right)(x \gamma) x^{\nu}\left[\tilde{F}_{\mu \nu}\left(\tau_{u} x\right)+i \gamma_{5} F_{\mu \nu}\left(\tau_{u} x\right)\right] \psi\left(\kappa_{2} u x\right), \\
S_{\mu}^{-}\left(\kappa_{1} x, \kappa_{2} x\right) & =\int_{0}^{1} d u \bar{u} \bar{\psi}\left(\kappa_{1} u x\right)(x \gamma) x^{\nu}\left[\tilde{F}_{\mu \nu}\left(\tau_{u} x\right)-i \gamma_{5} F_{\mu \nu}\left(\tau_{u} x\right)\right] \psi\left(\kappa_{2} u x\right),
\end{aligned}
$$

with $\tau_{u}=\bar{u} \kappa_{1}+u \kappa_{2}$; for notational simplicity we omitted the corresponding phase factors and the trace terms. Here, the Shuryak-Vainshtein operators $S^{ \pm}\left(\kappa_{1}, \tau_{u}, \kappa_{2}\right)$ in the integrand introduce additional complications because they are trilinear operators (being related to $O_{\mu}$ and $M_{\mu}$ by the equation of motion). As might be expected they are related to the symmetry class (ii) but with the following booking of indices

$$
\bar{\psi}(0) \gamma_{\left\{\mu_{1}\right.} D_{\mu_{2}} \ldots D_{\mu_{\ell-1}} D_{[\beta} D_{\left.\mu_{\ell}\right]} D_{\mu_{\ell}+1} \ldots D_{\left.\mu_{n}\right\}} \psi(0) .
$$

Therefore, these operators can be considered in the same manner as has been done for the vector (and tensor) operators of symmetry type (ii). A detailed treatment of this more evolved situation remained open.

\section{Acknowledgement}

The authors would like to thank Johannes Blümlein for many useful discussions. One of the authors (B.G.) grateful acknowledges the kind hospitality during a stay at Institute of Theoretical Physics, Karl-Franzens-University Graz, and another one (D.R.) thankful acknowledges a stay as guest of Graduate College "Quantum Field Theory" at Center for Higher Studies, Leipzig University. M.L. acknowledges the fine conditions at Max-Planck-Institute of Metal Research, Stuttgart, extended to him during the final stage of this work. Finally, we thank S. Serowy for a useful hint on the form of the LC-operators in axial gauge.

\footnotetext{
${ }^{13}$ In this Reference the trace terms had been ignored; in addition the equation of motion, $(i \gamma D-m) \psi=0$, has been used to relate the various possible twist-3 contributions which mix under renormalization
} 


\section{A Symmetry classes and tensor representations}

In this Appendix for the readers convenience we collect some facts about tensor representations of the classical matrix groups $G$ and their relation to the symmetric group $S_{n}$ which are of relevance in our consideration (see, e.g., [0, 9, 18, 29]). Given any irreducible matrix representation,

$$
G \ni g \mapsto \hat{g} \in \mathcal{B}(V): \quad \hat{g} e_{i}=e_{j} a_{i}^{j}
$$

on some vector space $V$ (of $\operatorname{dimension} \operatorname{dim} V$ ) with base $\left\{e_{i}, i=1,2, \ldots, \operatorname{dim} V\right\}$. The direct products of these representations act on the tensor space $T^{n} V$ which is build as tensor product of $n$ copies of the vector space $V$ :

$$
T^{n} V=(V \otimes \ldots \otimes V) .
$$

The elements of the tensor space $T^{n} V$ are the $n$-times contravariant tensors $\mathbf{t}=t^{i_{1} \ldots i_{n}} e_{i_{1} \ldots i_{n}}$, where $t^{i_{1} \ldots i_{n}}$ are the components of $\mathbf{t}$ in the base $e_{i_{1} \ldots i_{n}} \equiv\left(e_{i_{1}} \otimes\right.$ $\left.\ldots \otimes e_{i_{n}}\right), i_{k} \in[1, \ldots, \operatorname{dim} V]$. The components of the tensors of rank $n$ transform under $G$ according to

$$
\left(t^{\prime}\right)^{i_{1} \ldots i_{n}} \equiv t^{i_{1}^{\prime} \ldots i_{n}^{\prime}}=a^{i_{1}^{\prime}}{ }_{i_{1}} \ldots a^{i_{n}^{\prime}}{ }_{i_{n}} t^{i_{1} \ldots i_{n}}
$$

In the following we shall comment on the connection between the irreducible representations of the general linear group $G L(N, \mathbb{C})$ (together with its subgroups) and of the symmetric group $S_{n}$ on these tensor spaces. Let us, at the moment consider only the groups $G=G L(N, \mathbb{C}), S L(N, \mathbb{C}), S U(N)$. Given any (irreducible) representation of $G$ on $V$, then its $n$-fold tensor product defines a reducible representation $A^{(n)}$ which, together with the reducible representation $\hat{\pi}$ of $S_{n}$, is determined by:

$$
\begin{aligned}
& \hat{A}^{(n)}(g) \mathbf{t} \equiv \hat{A}^{(n)}(g)\left(t^{i_{1} \ldots i_{n}} e_{i_{1} \ldots i_{n}}\right):=t^{i_{1} \ldots i_{n}}(\hat{g} e)_{i_{1}} \otimes \ldots \otimes(\hat{g} e)_{i_{n}}, \quad \forall g \in G, \\
& \hat{\pi} \mathbf{t} \equiv \quad \hat{\pi}\left(t^{i_{1} \ldots i_{n}} e_{i_{1} \ldots i_{n}}\right):=t^{\pi\left(i_{1} \ldots i_{n}\right)} e_{i_{1}} \otimes \ldots \otimes e_{i_{n}}, \quad \forall \pi \in S_{n} ;
\end{aligned}
$$

therefore the action of both groups on the tensors $\mathbf{t}$ commutes

$$
\left[\hat{A}^{(n)}(g), \hat{\pi}\right] \mathbf{t}=0 \text {. }
$$

Of course, the same conclusion holds for the elements $\sum_{\pi} \alpha(\pi) \hat{\pi}, \alpha(\pi) \in \mathbb{R}$, of the group algebra $\mathcal{R}\left[S_{n}\right]$ of the symmetric group. This algebra, considered as a vector space, carries the regular representation of $S_{n}$ which is known to be fully reducible.

\footnotetext{
${ }^{14}$ Here, neither covariant tensors which act on direct products of the dual space $V^{*}$ nor mixed ones are considered since, for the case under consideration, the indices may be raised or lowered by the metric tensor.

${ }^{15}$ This results from the fact that the product of the matrix elements $a_{i}^{j}$ in eq. (A.2) is bisymmetric under $S_{n}$.
} 
The irreducible representations $\Delta^{[m]}$ of the symmetric group are uniquely determined by the idempotent (normalized) Young operators

$$
\begin{aligned}
\mathcal{Y}_{[m]} & =\frac{f_{[m]}}{n !} \mathcal{P} \mathcal{Q} \text { with } \mathcal{P}=\sum_{p \in H_{[m]}} p, \quad \mathcal{Q}=\sum_{q \in V_{[m]}} \delta_{q} q, \\
\mathcal{Y}_{[m]} \mathcal{Y}_{\left[m^{\prime}\right]} & =\delta_{[m]\left[m^{\prime}\right]} \mathcal{Y}_{[m]},
\end{aligned}
$$

which are related to corresponding Young tableaux being denoted by $[m]$. If

$$
\underline{m}=\left(m_{1}, m_{2}, \ldots m_{r}\right) \quad \text { with } \quad m_{1} \geq m_{2} \geq \ldots \geq m_{r}, \quad \sum_{i=1}^{r} m_{i}=n
$$

defines a Young pattern (Fig. 11) then a Young tableaux $[\mathrm{m}]$ is obtained by putting in (without repetition) the indices $i_{1}, \ldots i_{n}$ - corresponding to different "places" within the direct product - and $H_{[m]}$ and $V_{[m]}$ denotes their horizontal and vertical permutations with respect to $[\mathrm{m}]$. A standard tableaux is obtained when the indices $i_{1}, \ldots, i_{n}$ are ordered lexicographically. There are

$$
f_{[m]}=n ! \frac{\prod_{i<j}\left(l_{i}-l_{j}\right)}{\prod_{i=1}^{r} l_{i} !} \quad \text { with } \quad l_{i}=m_{i}+r-i, \quad \sum_{[m]} f_{[m]}^{2}=n !,
$$

different standard tableaux which correspond to $f_{[m]}$ different, but equivalent, irreducible representations of $S_{n}$ whose dimension is given also by $f_{[m]}$. The (normalized) Young operators $\mathcal{Y}_{[m]}$ according to (A.5) project onto mutually orthogonal irreducible left ideals of $\mathcal{R}\left[S_{n}\right]$.

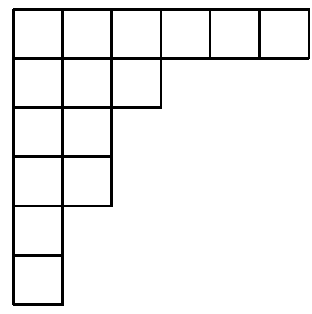

$$
\begin{aligned}
& m_{1} \text { boxes } \\
& m_{2} \text { boxes } \\
& \vdots \\
& m_{r} \text { boxes }
\end{aligned}
$$

Figure 1: Young pattern $\underline{m}$

In this way the tensor product of $n$ spaces $V$ carries the regular representation of the group $S_{n}$ and decomposes into $\sum_{[m]} f_{[m]}$ irreducible subspaces containing tensors of symmetry class $[m]$. However, the tensors actually are specified by taking for the indices $i_{1}, \ldots, i_{n}$ arbitrary values from the range $[1, \ldots, \operatorname{dim} V]$. Therefore, with respect to the group $G$ the standard tableaux is defined by putting into the Young pattern the values of the indices $i_{k}$ such that they are non-decreasing from left to right and increasing from top to bottom! Because of eq. (A.3) by Schur's Lemma it follows that the representation $\hat{A}^{(n)}(g)$ of $G$ is reducible and it decomposes into as many irreducible representations as there are (in general 
reducible) representations of $S_{n}$, i.e., any tensor representation of the group $G$ is characterized by a standard tableaux,

$$
\hat{A}^{(n)}(g)=\underset{[m]}{\oplus} R^{[m]}(g) \otimes E_{l_{[m]}}, \quad \hat{\pi}=\underset{[m]}{\bigoplus} E_{r_{[m]}} \otimes \Delta^{[m]}(\pi)
$$

where $E_{l_{[m]}}$ is the unit matrix; more explicitly we have

$$
\hat{A}^{(n)}(g)_{[m] \kappa \alpha,\left[m^{\prime}\right] \kappa^{\prime} \alpha^{\prime}}=\delta_{[m]\left[m^{\prime}\right]} \delta_{\alpha \alpha^{\prime}} R_{\kappa \kappa^{\prime}}^{[m]}(g)
$$

where $\kappa=1, \ldots, r_{[m]}$ counts different equivalent irreducible representations of $S_{n}$ and $\alpha=1, \ldots, l_{[m]} \leq f_{[m]}$ counts the basis elements of these representations. The representations $R^{[m]}(g)$ of $G$ in $T^{n} V$ are characterized by equivalent Young operators $\widehat{\mathcal{Y}}_{[m]}=\mathcal{Q P}:$ 田

$$
T^{n} V=\underset{[m] \kappa}{\oplus} \widehat{\mathcal{Y}}_{[m] \kappa}\left(T^{n} V\right)
$$

The invariant subspaces projected out by $\widehat{\mathcal{Y}}_{[m] \kappa}\left(T^{n} V\right)$ are irreducible with respect to $G L(N, \mathbb{C}), S L(N, \mathbb{C})$ and $S U(N)$. After restriction onto these subgroups some of the irreducible representations become equivalent ones. However, for $O(N, \mathbb{C}), S O(N, \mathbb{C})$ and $S p(2 \nu)$ these representations, in general, are not irreducible and decompose further.

Concerning the orthogonal groups from the subspaces of the above introduced symmetry classes only the completely antisymmetric ones (for $n \leq N$ ) remain irreducible. The reason is that, because of the very definition of the orthogonal groups, $\delta_{i j} a_{k}^{i} a^{j}{ }_{l}=\delta_{k l}, \forall a \in O(N)$, the operation of taking the trace of a tensor commutes with the orthogonal transformations of that tensor:

$$
\operatorname{tr} \mathbf{T}^{\prime}=\delta_{i j} T^{\prime i j}=\delta_{i j} a_{k}^{i} a^{j}{ }_{l} T^{k l}=\operatorname{tr} \mathbf{T} .
$$

Again, by Schur's Lemma, irreducible subspaces of $O(N)$ are spanned by traceless tensors having definite symmetry class. This decomposition is obtained as follows:

$$
T_{[m]}^{i_{1} i_{2} \ldots i_{n}}=\stackrel{\circ}{T_{[m]}^{i_{1} i_{2} \ldots i_{n}}}+\sum_{1 \leq r, s \leq n} \delta^{i_{r} i_{s}} T_{[m-2]}^{i_{1} \ldots i_{r-1} i_{r+1} \ldots i_{s-1} i_{s+1} \ldots i_{n}} .
$$

The tensors which appear under the sum have degree $n-2$ and a Young pattern $[m-2] \subset[m]$ obtained by removing two boxes from the (right) border without destroying the property $(2.2)$ and $(2.3)$ to be a pattern. They may be decomposed again into traceless ones plus some remainder, and so on. Therefore, a traceless tensor is obtained from the original one by succesively subtracting the traces.

\footnotetext{
${ }^{16}$ Note, that the symmetrizations $\mathcal{P}$ and the antisymmetrizations $\mathcal{Q}$ with respect to $[m]$ are interchanged. This corresponds, in the terminology of quantum mechanics, to "quantum number permutations" instead of the "place permutations" above. And, contrary to $\mathcal{Y}_{[m]}$ which defines a left ideal in $\mathcal{R}, \widehat{\mathcal{Y}}_{[m]}$ defines a right ideal.
} 


\section{B Harmonic tensor functions}

In this Appendix we derive the projection operators which determine the traceless part of a completely symmetric tensor of rank $n, T_{\left\{\mu_{1} \ldots \mu_{n}\right\}}$, of a tensor of rank $n+1$ being symmetric in $n$ of its indices, $T_{\alpha\left\{\mu_{1} \ldots \mu_{n}\right\}}$, and of a tensor of rank $n+2$ being symmetric in $n$ and antisymmetric in the remaining two indices, $T_{[\alpha \beta]\left\{\mu_{1} \ldots \mu_{n}\right\}}$. To achive this we use the fact that, after contracting the indices of the symmetric part with some vector $x$, the resulting scalar, vector and antisymmetric tensor functions obey the following equations:

$$
\begin{array}{rlrl}
\square \stackrel{\circ}{T}_{n}(x) & =0, & \\
\square \stackrel{\circ}{T}_{\alpha n}(x) & =0, & \partial^{\alpha} \stackrel{\circ}{T}_{\alpha n}(x) & =0, \\
\square \stackrel{\circ}{T}_{[\alpha \beta] n}(x) & =0, & \partial^{\alpha} \stackrel{\circ}{T}_{[\alpha \beta] n}(x) & =0,
\end{array}
$$

(i) The first of these equations define the harmonic polynomials of order $n$. Its solution, in $d$ dimensions, is given by (see e.g. [32], Chapter IX)

$$
\begin{aligned}
\stackrel{\circ}{T}_{n}(x) & =H_{n}^{(d)}\left(x^{2} \mid \square\right) T_{n}(x) \\
& =\left\{1+\sum_{k=1}^{\left[\frac{n}{2}\right]}\left(\prod_{\ell=1}^{k} \frac{1}{d+2 n-2 \ell-2}\right) \frac{\left(-x^{2}\right)^{k} \square^{k}}{2^{k} k !}\right\} T_{n}(x),
\end{aligned}
$$

which, for $d=4$, may be rewritten as

$$
\stackrel{\circ}{T}_{n}(x)=H_{n}^{(4)}\left(x^{2} \mid \square\right) T_{n}(x)
$$

with the harmonic projection operator

$$
H_{n}^{(4)}\left(x^{2} \mid \square\right)=\sum_{k=0}^{\left[\frac{n}{2}\right]} \frac{(n-k) !}{k ! n !}\left(\frac{-x^{2}}{4}\right)^{k} \square^{k} .
$$

(ii) Let us now consider the set of equations $(\mathbb{B} .2)$ to determine the harmonic vector functions $T_{\alpha n}(x)$ being related to the traceless tensors $T_{\alpha\left\{\mu_{1} \ldots \mu_{n}\right\}}$ which, to the best of our knowledge, are not determined up to now. Of course, after the $n$-fold contraction with $x$ they are harmonic polynomials of order $n$,

$$
T_{\alpha \stackrel{\circ}{\circ}}(x):=H_{n}^{(4)}\left(x^{2} \mid \square\right) T_{\alpha n}(x)
$$

and the traces corresponding to $g_{\alpha \mu_{i}}$ are obtained through differentiation with respect to $x_{\alpha}$. Therefore, the most general ansatz for the harmonic vector functions which conserves its order is

$$
\stackrel{\circ}{T}_{\alpha n}(x)=\left\{\delta_{\alpha}^{\beta}-a_{n} x_{\alpha} \partial^{\beta}(x \partial)-b_{n} x^{2} \partial_{\alpha} \partial^{\beta}\right\} H_{n}^{(4)}\left(x^{2} \mid \square\right) T_{\beta n}(x) .
$$


From the two conditions $(\mathbb{B} .2)$ we obtain two equations from which the coefficients $a_{n}, b_{n}$ can be determined:

$$
\begin{aligned}
& 1=n(d+n-1) a_{n}+2(n-1) b_{n} \\
& 0=n a_{n}+(d+2 n-4) b_{n}
\end{aligned}
$$

Note, that to derive these conditions we used the fact that $\square H_{n}^{(4)}\left(x^{2} \mid \square\right) \equiv 0$, $\left[(x \partial), H_{n}^{(4)}\right]=0$ and $(x \partial) T_{\beta n}(x)=n T_{\beta n}(x)$. The solution of these equations is

$$
n a_{n}=\frac{d+2 n-4}{(d+2 n-2)(d+n-3)}, \quad b_{n}=-\frac{1}{(d+2 n-2)(d+n-3)} ;
$$

for $d=4$ it reduces to

$$
a_{n}=1 /(n+1)^{2}, \quad b_{n}=-a_{n} / 2 .
$$

Therefore, we obtain the following result:

$$
\stackrel{\circ}{T}_{\alpha n}(x)=\left\{\delta_{\alpha}^{\beta}-\frac{1}{(n+1)^{2}}\left[x_{\alpha} \partial^{\beta}(x \partial)-\frac{1}{2} x^{2} \partial_{\alpha} \partial^{\beta}\right]\right\} H_{n}^{(4)}\left(x^{2} \mid \square\right) T_{\beta n}(x) .
$$

If eq. (B.9) will be contracted with $x^{\alpha}$ then, after some tedious but straightforward calculations, we obtain, as is should be, the harmonic polynomial $\stackrel{\circ}{T}_{n+1}(x)$.

(iii) Using the same procedure we may construct the harmonic antisymmetric tensor functions $\stackrel{\circ}{T}_{[\alpha \beta] n}(x)$. The most general ansatz for it has the following structure (remind the convention $T_{[\mu \nu]}=\frac{1}{2}\left(T_{\mu \nu}-T_{\nu \mu}\right)$ ):

$$
\stackrel{\circ}{T}_{[\alpha \beta] n}=\left\{\delta_{\alpha}^{\mu} \delta_{\beta}^{\nu}-a_{n} x_{[\alpha} \delta_{\beta]}^{\nu} \partial^{\mu}-b_{n} x^{2} \partial^{\mu} \partial_{[\alpha} \delta_{\beta]}^{\nu}-c_{n} x^{\mu} x_{[\alpha} \partial_{\beta]} \partial^{\nu}\right\} T_{[\mu \nu] n}(x) .
$$

The conditions (B.3) lead to the requirement that the coefficients of the terms $\delta_{\beta}^{\mu} \partial^{\nu}, x^{\mu} \partial^{\nu} \partial_{\beta}$ and $\partial_{[\alpha} \delta_{\beta]}^{\mu} \partial^{\nu}$ should vanish. The corresponding system of linear equations reads

$$
\begin{aligned}
& 1=(d+n-2) a_{n} / 2+(n-1) b_{n} \\
& 0=2 b_{n}+(d+n-2) c_{n} \\
& 0=a_{n}+c_{n}+(d+2 n-4) b_{n}
\end{aligned}
$$

It is solved by the following values:

$$
\left(a_{n}+c_{n}, b_{n}, c_{n}\right)=\frac{2}{(d+2 n-2)(d+n-4)}\left(d+2 n-4,-1, \frac{2}{(d+n-2)}\right),
$$

and for $d=4$ we obtain

$$
\left(a_{n}+c_{n}, b_{n}, c_{n}\right)=\frac{2}{n(n+1)(n+2)}\left(n(n+2),-\frac{1}{2}(n+2), 1\right) .
$$


From this, using the replacement $x^{\mu} x_{[\alpha} \partial_{\beta]} \partial^{\nu}=x_{[\alpha} \partial_{\beta]} x^{\mu} \partial^{\nu}-x_{[\alpha} \delta_{\beta]}^{\mu} \partial^{\nu}$ we finally obtain:

$$
\begin{aligned}
\stackrel{\circ}{T}_{[\alpha \beta] n}(x)= & \left\{\delta_{[\alpha}^{\mu} \delta_{\beta]}^{\nu}-\frac{2}{n(n+1)(n+2)}\left(x_{[\alpha} \partial_{\beta]} x^{[\mu} \partial^{\nu]}\right.\right. \\
& \left.\left.-\left[x_{[\alpha} \delta_{\beta]}^{[\mu} \partial^{\nu]}(x \partial)-\frac{1}{2} x^{2} \partial_{[\alpha} \delta_{\beta]}^{[\mu} \partial^{\nu]}\right](x \partial+2)\right)\right\} H_{n}^{(4)}\left(x^{2} \mid \square\right) T_{[\mu \nu] n}(x) .
\end{aligned}
$$

Let us remark that the various differential operators which appear in eqs. (B.9) and (B.11) may be expressed by the generators of the conformal group, namely $P_{\mu}, M_{\mu \nu}, D$ and $K_{\mu}$.

\section{References}

[1] M.A. Ahmed and G.G. Ross, Nucl. Phys. B 111(1976) 441;

Phys. Lett. 56 B(1975) 385

[2] S.A. Anikin and O.I. Zavialov, Ann. Phys. (NY) 116 (1978) 135;

O.I. Zavialov, Renormalized Feynman Diagrams, (Nauka, Moscow, 1979), in Russian; Renormalized Quantum Field Theory (Kluwer Academic Press, Dordrecht, 1990), extended English translation.

[3] I.I. Balitsky, Phys. Lett. 124B (1983) 230;

[4] I.I. Balitsky and V.M. Braun, Nucl. Phys. B311 (1988/89) 541

[5] I.I. Balitsky, V.M. Braun, and A.V.Kolesnichenko, Nucl. Phys. B312 (1989) 509

[6] P. Ball, V.M. Braun, Y. Koike, and K. Tanaka, Nucl. Phys. B529 (1998) 323

[7] A.O. Barut and R. Raczka, Theory of Group Representations and Applications PWN - Polish Scientific Publishers, Warszawa, 1977

[8] J. Blümlein, B. Geyer, and D. Robaschik, Virtual Compton scattering in the generalized Bjorken region: The Twist-2 case, to appear hep-ph/9903520

[9] H. Boerner: Darstellungen von Gruppen, Springer-Verlag, Berlin (1955)

[10] M. Bordag, B. Geyer, J. Hořejši, and D. Robaschik, Zs. Phys. C26 (1985) 591 ;

[11] T. Braunschweig, B. Geyer, J. Hořejši, and D. Robaschik, Zs. Phys. C33 (1987) 275;

[12] M. Bordag and D. Robaschik, Nucl. Phys. B169 (1980) 445;

B. Geyer, D. Müller, and D. Robaschik, preprint SLAC-Pub 1993; 
[13] A.P. Bukhvostov, E.A. Kuraev, and L.N. Lipatov, Jad. Fiz. 38 (1983) 439 [Sov. J. Nucl. Phys. 38 (1983) 263]; Jad. Fiz. 39 (1984) 194 [Sov. J. Nucl. Phys. 39 (1984) 121]

[14] V.K. Dobrev, A.Ch. Ganchev, and O.I. Yordanov, Phys. Lett. 119B (1982) 372

V.K. Dobrev and A.Ch. Ganchev, Conformal operators from spinor fields: Antisymmetric tensor case, Dubna preprint E2-82-881 (1982)

[15] B. Geyer, D. Müller, and D. Robaschik, Nucl. Phys. B (Proc. Suppl.) 51 C (1996) 106, hep-ph/9611452

[16] D.J. Gross and S.B. Treiman, Phys. Rev. D 4 (1971) 1059

[17] P.A.M. Guichon and M. Vanderhaeghen, Virtual Compton Scattering off the nucleon, Prog. Part. Nucl. Phys. 41 (1998) xxx, hep-ph/9806305

[18] M. Hamermesh, Group theory and its application to physical problems, AddisonWesley, London (1962)

[19] R.L. Jaffe and X. Ji, Phys. Rev.D43 (1991) 724

[20] R. Jaffe and X. Ji, Nucl. Phys. B375 (1992) 527;

X. Ji, Nucl. Phys. B402 (1993) 217

[21] R.L. Jaffe: Spin, twist and hadron structure in deep inelastic processes, Erice Lectures, hep-ph/9602236

[22] X. Ji, Phys. Rev. Lett. 78 (1997) 610 [hep-ph/9609381];

Phys. Rev. D55 (1997) 7114.

[23] J. Kodaira, S. Matsuda, K. Sasaki, and T. Uematsu, Nucl. Phys. B 159 (1979) 99

[24] K. Kogut and D.E. Soper, Phys. Rev. D1 (1970) 2901

[25] Y. Koike and K. Tanaka, Phys. Rev. D 51 (1995) 6125-6138

[26] M. Lazar, Konstruktion und Anwendung irreduzibler nichtlokaler Lichtkegeloperatoren in der Quantenchromodynamik, Diplomarbeit, Leipzig 1998

[27] D. Müller, D. Robaschik, B. Geyer, F.-M. Dittes, and J. Hořejši: Wave functions, evolution equations and evolution kernels from light-ray operators of QCD, Fortsch. Phys. 42 (1994) 101;

reprinted as hep-ph/9812448

[28] O. Nachtmann, Nucl. Phys. B 63 (1973) 237 
[29] M. I. Petraschen and E. D. Trifonov, Anwendungen der Gruppentheorie in der Quantenmechanik, Moscow, Nauka 1967 (in Russian), translated into German, Berlin, Dt. Verlag d. Wissenschaften, 1969

[30] A.V. Radyushkin, Phys. Lett. B380 (1996) 417; Phys. Rev. D56 5524; Phys. Lett. B380 (1996) 417 [hep-ph/9704207].

[31] E.V. Shuryak, A.I. Vainshtein, Nucl. Phys. B 201 (1982) 141

[32] N.Ya. Vilenkin, Special functions and the theory of group representations Nauka, Moscow 1965 (in Russian),

N.Ya. Vilenkin and A.U. Klimyk, Representations of Lie groups and Special Functions, Vol. 2., Kluwer Academic Publishers, Dordrecht 1993 